\title{
Discontinuity of Hausdorff dimension and limit capacity on arcs of diffeomorphisms
}

\author{
LORENZO J. DIAZ \\ IMPA, Estrada Dona Castorina 110, 22.460-Rio de Janeiro-RJ, Brasil \\ AND \\ MARCELO VIANA $\dagger$ \\ Departamento de Matemática, Fac. Ciências do Porto, 4000 Porto-Portugal
}

(Received 27 April 1988 and revised 21 February 1989)

\begin{abstract}
We consider one-parameter families of torus diffeomorphisms that bifurcate from global hyperbolic maps (Anosov) to DA maps (derived from Anosov). For an open set of these families, we show that the Hausdorff dimension and limit capacity of the nonwandering set are not continuous across the bifurcation. We also study the behaviour of equilibrium measures near the bifurcation.
\end{abstract}

\section{Introduction}

In [10] Smale showed that global hyperbolic (Anosov) diffeomorphisms on the torus can be modified to get Axiom A diffeomorphisms whose nonwandering set consists of a fixed source and a one-dimensional attractor (DA maps). It is a natural question to ask if the dimension of the nonwandering set varies continuously during the process of construction of these DA diffeomorphisms. In [4] McCluskey and Manning gave an affirmative answer to this question; however their proof is incorrect as pointed out by Manning in [6]. In fact we show here that the result itself is false: in general just the opposite is true. We prove for an open family of arcs of $C^{2}$. diffeomorphisms from Anosov to DA with a nondegenerate saddle-node bifurcation, that the Hausdorff dimension and limit capacity are discontinuous, since at the bifurcation value they are strictly smaller than 2 . On the other hand, we can also show that these dimensions may have continuous variations across a degenerate bifurcation from Anosov to DA maps. Another interesting question ([2, Chapter 6]) is to describe the behaviour of equilibrium states of DA maps near the bifurcation. We prove that for arcs with generic saddle-node bifurcations as above the equilibrium states of $\log \left|D f_{t}\right|_{E_{1}^{s}} \mid$ (where $E_{t}^{s}$ denotes the stable bundle of $f_{t}$ ) converge to the Dirac measure supported at the saddle-node.

Acknowledgements. We would like to express our gratitude to our supervisor Professor J. Palis for introducing to us the subject and proposing this problem as well as for his constant encouragement during the preparation of this paper. We also

† Current address: IMPA, Estrada Dona Castorina 110, 22.460-Rio de Janeiro-RJ, Brasil. 
thank useful comments by G. Contreras. The first author acknowledges partial support from Research Project CAICyT/S.D. 84-397 (Spain).

\section{Basic definitions and statement of results}

Let $M$ be a compact surface. We recall some results on Hausdorff dimension and limit capacity of basic sets of diffeomorphisms on $\boldsymbol{M}$. For Hausdorff dimension these results are mostly due to McCluskey and Manning [4]. Elementary proofs and further informations were provided in [8]. See Takens [11] for the relation between Hausdorff dimension and limit capacity.

For $X$ a metric space and $\alpha>0$ we define the $\alpha$-measure of $X$ by

$$
m_{\alpha}(X)=\sup _{\varepsilon>0} \inf _{q \ell}\left(m_{\alpha}(\mathcal{U})\right)
$$

where

$$
m_{\alpha}(\mathscr{U})=\sum_{U \in \mathscr{U}}(\operatorname{diam}(U))^{\alpha}
$$

and the infimum is taken over all countable covers of $X$ by sets with diameter less than $\varepsilon$. It is not hard to see that there is a unique number, the Hausdorff dimension of $X$, denoted by $H D(X)$, such that, for $\alpha<H D(X), m_{\alpha}(X)=\infty$, and, for $\alpha>H D(X), m_{\alpha}(X)=0$.

The limit capacity of $X$ is defined by

$$
d(X)=\lim \sup _{\varepsilon \rightarrow 0} \log n(X, \varepsilon) / \log \varepsilon^{-1},
$$

where $n(X, \varepsilon)$ is the minimum number of $\varepsilon$-balls that cover $X$.

It is easy to see that both Hausdorff dimension and limit capacity are invariant by Lipschitz homeomorphisms and that $d(X) \geq H D(X)$ for all $X$.

Recall that a compact $\Lambda \subset M$ is a basic set for a diffeomorphism $f$ of $M$ if it is invariant, hyperbolic, transitive, has a dense subset of periodic orbits and it is the maximal invariant set for $f$ in a neighbourhood $U$ of $\Lambda$ ([3]). For a basic set $\Lambda$ the values of $H D\left(\Lambda \cap W^{s}(x, f)\right)$ and $d\left(\Lambda \cap W^{s}(x, f)\right)$ are independent of $x \in \Lambda$ and called stable Hausdorff dimension $\left(H D^{s}(\Lambda)\right)$ and stable limit capacity $\left(d^{s}(\Lambda)\right)$ of $\Lambda$, respectively. Dually we define $H D^{u}(\Lambda)$ and $d^{u}(\Lambda)$. In this case

and

$$
H D^{i}(\Lambda)=d^{i}(\Lambda), \quad i=s, u,
$$

$$
H D(\Lambda)=H D^{s}(\Lambda)+H D^{u}(\Lambda)=d^{s}(\Lambda)+d^{u}(\Lambda)=d(\Lambda) .
$$

Moreover these invariants depend continuously on the diffeormorphism in the sense that $H D^{i}\left(\Lambda_{\mathrm{g}}\right)$ and $d^{i}\left(\Lambda_{\mathrm{g}}\right), i=s, u$, are continuous functions of $g$ (in the $C^{1}$ topology) where $\Lambda_{g}=\bigcap_{n} g^{n}(U)$ is the analytical continuation of $\Lambda$ for $g$ close to $f$.

We now sketch the construction of DA arcs (see Williams [13] for details). Let $G \in G l(Z, 2)$ have eigenvalues $\lambda$ and $\lambda^{-1}, 0<\lambda<1<\lambda^{-1}$, and $g$ be the Anosov diffeomorphism on $T^{2}=\mathbf{R}^{2} / Z^{2}$ induced by $G$. Denote by $\theta$ the fixed point of $g$.

We deform $g$ by isotopy to construct an $\operatorname{arc}\left(f_{t}\right)$, of $C^{2}$ diffeomorphisms such that (see figure 1):

(1) $f_{-1}=g$ and $f_{t}$ is Anosov for $t<0$ (we denote $\Lambda_{t}=T^{2}$ ) 

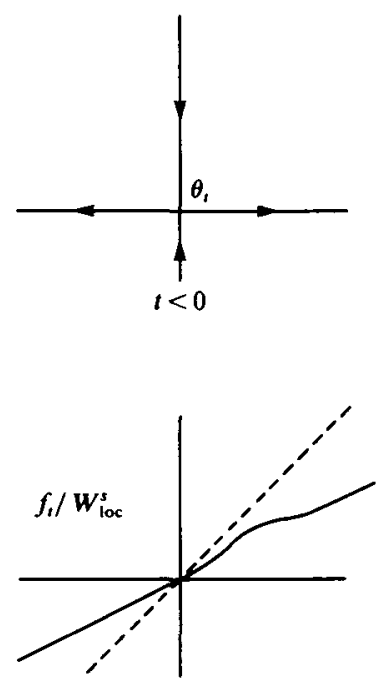
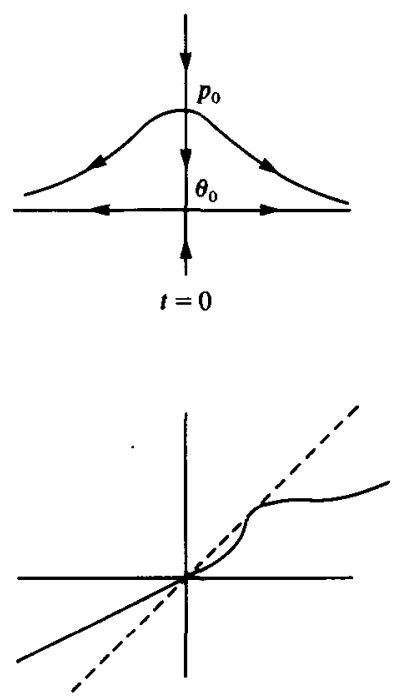

Figure 1
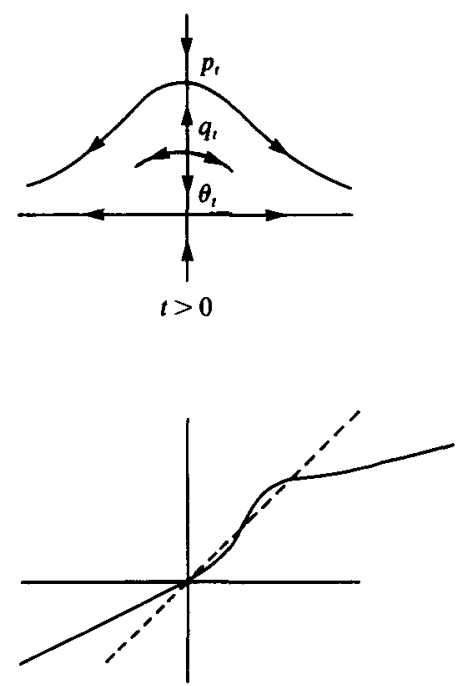

(2) $f_{0}$ has a fixed quadratic saddle-node point $p_{0}$, near $\theta$, and $T^{2}=\operatorname{int}\left(W^{u}\left(p_{0}\right)\right) \cup \Lambda_{0}$ with $\Lambda_{0}$ compact, invariant and transitive

(3) for $t>0$ the saddle-node splits into two fixed points, a source $q_{t}$ and a saddle $p_{t} ; f_{t}$ is Axiom $A, T^{2}=W^{u}\left(q_{t}\right) \cup \Lambda_{t}$ and $\Omega\left(f_{t}\right)=\left\{q_{t}\right\} \cup \Lambda_{t}$, where $\Lambda_{t}$ is a onedimensional hyperbolic attractor, locally homeomorphic to the product of a Cantor set and an interval.

For Theorem A we need some technical hypothesis whose statement is postponed to $\S 2$. We point out that these hypotheses are satisfied by an open set of arcs of diffeomorphisms.

THEOREM A. Let $\left(f_{t}\right)$, be an arc of $C^{2}$-diffeomorphisms as above, satisfying conditions (C1)-(C5) of \&2. Then:

(a) $\frac{3}{2}<H D\left(\Lambda_{0}\right)<2$,

(b) $\lim _{t \rightarrow 0^{+}} H D\left(\Lambda_{t}\right)=H D\left(\Lambda_{0}\right)$,

(c) $H D\left(\Lambda_{0}\right)=d\left(\Lambda_{0}\right)$.

Since $H D\left(\Lambda_{t}\right)=2$ for $t<0$ the upper bound in (a) implies that $H D\left(\Lambda_{t}\right)$ is not continuous at $t=0$, although by $(b)$, it is continuous on the right. As $d\left(\Lambda_{t}\right)=H D\left(\Lambda_{t}\right)$ for all $t \neq 0$ (see above) (c) implies analogous facts for the limit capacity.

We now consider a different construction of DA maps. Let $g$ be deformed by isotopy through an arc of $C^{2}$-diffeomorphisms $\left(f_{t}\right)$, such that:

(1) $f_{-1}=g$ and $f_{t}$ is Anosov for $t<0$.

(2) For $f_{0}$ there is a nonhyperbolic fixed saddle point $\theta_{0}$ near $\theta$, with eigenvalue 1 , and $\Omega\left(f_{0}\right)=T^{2}$.

(3) For $t>0, \theta_{0}$ splits into three fixed points: a source $\theta_{t}$ and two saddles $p_{t}$ and $q_{t} ; f_{t}$ is Axiom $\mathrm{A}, T^{2}=\Lambda_{t} \cup W^{u}\left(\theta_{t}\right)$ and $\Omega\left(f_{t}\right)=\Lambda_{t} \cup\left\{\theta_{t}\right\}, \Lambda_{t}$ being a hyperbolic attractor.

In this case we state: 
THEOREM B. Let $\left(f_{t}\right)$, be an arc of $C^{2}$-diffeomorphisms as above satisfying conditions (C1)-(C5) of $\$ 2$. Then:

$$
\lim _{t \rightarrow 0^{+}} H D\left(A_{t}\right)=\lim _{t \rightarrow 0^{+}} d\left(\Lambda_{t}\right)=2 .
$$

Since $H D\left(\Lambda_{t}\right)=d\left(\Lambda_{t}\right)=2$ for $t \leq 0$, it follows immediately from Theorem B that the Hausdorff dimension and the limit capacity of the non-wandering set are continuous along the arc.

Let $\Lambda$ be a compact metric space and $f: \Lambda \rightarrow \Lambda$ an expansive map. The pressure of a continuous real function $\varphi: \Lambda \rightarrow R$ is defined by

$$
P(\varphi)=\limsup _{n \rightarrow \infty} \frac{1}{n} \log \sup \sum_{x \in E} \exp \left(\sum_{0}^{n-1} \varphi\left(f^{i}(x)\right),\right.
$$

where the supremum is taken over $(n, \delta)$-separated sets $E$ and $\delta$ is an expansive constant for $\left.f\right|_{\Lambda}$. By the variational principle (Walters [12])

$$
P(\varphi)=\sup _{\mu}\left(h_{\mu}(f)+\int \varphi d_{\mu}\right)
$$

where the supremum is taken over $f$-invariant Borel probability measures. An equilibrium state for $\varphi$ is an $f$-invariant measure $\mu$ such that $P(\varphi)=h_{\mu}(f)+\int \varphi d_{\mu}$. If $\Lambda$ is a basic set of an Axiom A diffeomorphism $f$, then equilibrium states exist and are unique for all Hölder continuous functions (Bowen [1]).

Let $\phi_{t}^{s}=\log \left|D^{s} f_{t}\right|$, where $D^{s} f_{t}$ denotes the derivative of $f_{t}$ in the stable direction. Theorem A allows us to give a description of the behaviour of the equilibrium states of $\phi_{t}^{s}$ near the bifurcation.

Theorem C. Let $\left(f_{t}\right)_{t}$ be as in Theorem A and $\mu_{t}$ be the equilibrium state for $\phi_{t}^{s}$. Then $\mu_{t} \rightarrow \delta$ as $t \rightarrow 0^{+}$, where $\delta$ is the Dirac measure supported at the saddle-node. Moreover $\delta$ is the unique equilibrium state for $\phi_{0}^{s}$.

These results are proved in the sections below. In order to simplify the notations we will in what follows omit the subscript $t=0$ (and so write $f$ for $f_{0}, p$ for $p_{0}$, $\Lambda$ for $\Lambda_{0}$, etc), except where dependence on $t$ is directly involved.

\section{The unstable foliation}

If $\Omega$ is a basic set of a $C^{2}$ diffeomorphism on a surface then (Hirsch and Pugh [3]) the unstable foliation $\mathscr{F}^{\mu}$ of $\Omega$, defined by $\mathscr{F}_{x}^{u}=W^{u}(x)$ for $x \in \Omega$, is differentiable in the sense that its holonomy projections can be extended to $C^{1}$ mappings. This is still true for the non-hyperbolic attractor $\Lambda=\Lambda_{0}$. In fact we prove somewhat more:

Proposition 2.1. Under conditions (C1)-(C5) below, there is an f-invariant $C^{1}$ foliation $\mathscr{F}^{u}$ of $T^{2}$ whose leaves are uniformly expanded by $f$ and such that $\Lambda$ is $\mathscr{F}^{u}$-invariant (i.e. it is a union of $\mathscr{F}^{u}$-leaves).

Proof. The proposition follows from essentially the same argument as the hyperbolic case, so we only sketch here the main ideas. Consider (local) coordinates $(\xi, \eta)$ on $T^{2}$ such that 


$$
\left.\frac{\partial}{\partial \xi}\right|_{\pi(x)}=D \pi(x) \cdot v^{u} \text { and }\left.\frac{\partial}{\partial \eta}\right|_{\pi(x)}=D \pi(x) \cdot v^{s} \quad \text { for all } x \in \mathbf{R}^{2},
$$

where $v^{u}$ (resp. $v^{s}$ ) is some expanding (resp. contracting) eigenvector of $G$. Take the Riemann metric on the torus for which $\left\{\partial /\left.\partial \xi\right|_{z}, \partial /\left.\partial \eta\right|_{z}\right\}$ is an orthonormal basis of $T_{z}\left(T^{2}\right)$ for all $z \in T^{2}$ (for our purposes it does not matter which Riemann metric we use since they are all Lipschitz equivalent). Let $f(\xi, \eta)=(\phi(\xi, \eta), \psi(\xi, \eta))$ be the local representation of $f$. We assume that

(C1) $\left|\phi_{\xi}^{-1}\right|<\lambda+\varepsilon$,

(C2) $\left|\phi_{\eta}\right|<\varepsilon$,

(C3) $\left|\psi_{\xi}\right|<\varepsilon$,

(C4) $\left|\psi_{\eta}\right|<(1+\varepsilon)$,

(C5) $\left|\psi_{\eta}\right|<\lambda+\varepsilon$ outside a small neighbourhood $Q$ of the non-hyperbolic fixed point $p$ (resp. $\theta_{0}$ in Theorem B),

where $\varepsilon>0$ is such that $(1+\varepsilon) \cdot(\lambda+\varepsilon)<1$. Then (minor additional restrictions on the value of $\varepsilon>0$ may still be needed), the methods of [3] can be used to construct a 1-dimensional, $C^{1}$ vector bundle on $T^{2}, E^{u}$, such that $\left.D f\right|_{E^{u}}$ is uniformly expanding. Let $\mathscr{F}^{u}$ be the integral foliation of $E^{u}$. Since int $\left(W^{u}(p)\right)$ is $\mathscr{F}^{u}$-invariant $\left(f^{-1}\right.$ is contracting on the leaves of $\mathscr{F}^{u}$, and moreover $\left.\mathscr{F}^{u}(p)=W^{u u}(p)\right)$, the same holds for $\Lambda=\left(\text { int }\left(W^{u}(p)\right)\right)^{c}$. This ends the proof of the proposition.

Remark 2.2. In particular, projections along the leaves of $\mathscr{F}_{0}^{u}=\mathscr{F}^{u}$ are Lipschitz continuous. Now, conditions (C1)-(C5) persist for small perturbations of $f=f_{0}$, so the arguments above also yield the unstable foliation $\mathscr{F}_{t}^{u}$ of $\Lambda_{t}$, for all small values of $t$. Moreover, by the uniformity of the construction we get that Lipschitz constants for the holonomy maps of $\mathscr{F}_{t}^{u}$ may be taken independent of $t$ (small). We will use this fact in $\$ 6$ and $\$ 7$.

From the proposition (and the remark) it follows that $\Lambda_{t}$ is locally Lipschitz homeomorphic to $\mathscr{F}_{t}^{u}(x) \times\left(\sum_{x} \cap \Lambda_{t}\right)$, where $\sum_{x}$ is any (small) section transversal to $\mathscr{F}^{u}$, at $x \in \Lambda_{t}$. Then

$$
d\left(\Lambda_{t}\right) \leq d\left(\mathscr{F}_{t}^{u}(x)\right)+d\left(\sum_{x} \cap \Lambda_{t}\right)=1+\left(\sum_{x} \cap \Lambda_{t}\right)
$$

and (Marstrand [7])

$$
H D\left(\Lambda_{t}\right) \geq 1+H D\left(\Sigma_{x} \cap \Lambda_{t}\right)
$$

On the other hand, again by Proposition 2.1 (see [8] for example), $d\left(\sum_{x} \cap \Lambda_{t}\right)$ and $H D\left(\sum_{x} \cap \Lambda_{t}\right)$ are independent of $\sum_{x}$ and $x \in \Lambda_{t}$. We denote their values by $d^{s}\left(\Lambda_{t}\right)$ and $H D^{s}\left(\Lambda_{t}\right)$ respectively. Since $H D\left(\Lambda_{t}\right) \leq d\left(\Lambda_{t}\right)$ for all $t$ this reduces the proof of Theorems A and B to proving, respectively,

THEOREM A'. Under the hypothesis of Theorem A

(a) $\frac{1}{2}<H D^{s}\left(\Lambda_{0}\right)<1$,

(b) $H D^{s}\left(\Lambda_{t}\right) \rightarrow H D^{s}\left(\Lambda_{0}\right)$ as $t \rightarrow 0^{+}$,

(c) $H D^{s}\left(\Lambda_{0}\right)=d^{s}\left(\Lambda_{0}\right)$. 
THEOREM B'. Under the hypothesis of Theorem B

$$
H D^{s}\left(\Lambda_{t}\right) \rightarrow 1 \text { as } t \rightarrow 0^{+} \text {. }
$$

We end this section by showing that $f$ is uniformly contractive on $W^{s}(p)$, outside some small neighbourhood $Q$ of $p$ (recall (C5)). Note first that, if $\varepsilon>0$ is small, then $T_{z} W^{s}(p)$ must be almost vertical (relative to coordinates $(\xi, \eta)$ ) at all point $z \in W^{s}(p)$. In fact, suppose that for some $z \in W^{s}(p)$, the angle between $T_{z} W^{s}(p)$ and the $\eta$-direction were not small. Then, by conditions (C1)-(C5), it would be increased by $D f^{n}$ and we would get $T_{p} W^{s}(p)=\lim _{n} T_{f^{n}(z)} W^{s}(p)$ nearly horizontal. This is absurd because $D f$ expands in the $\xi$-direction. So that angle must be (uniformly) small. Now, conditions (C1)-(C5) (specially (C5)), imply that $\left|D^{s} f(z)\right| \leq$ $\lambda_{0}$, for all $z \in W^{s}(p)-Q$, where $\lambda<\lambda_{0}<1$ and $D^{s} f(z)=\left.D f\right|_{T_{z} W^{s}(p)}$. This is what we wanted to prove.

\section{3. $H D^{s}\left(\Lambda_{0}\right)<1$}

First we introduce some notations. In what follows $<$ is the natural ordering in $W=W^{s}(p)-\{p\}$ such that $f(x)<x$ for all $x \in W$. Denote the length of a connected subset $I$ of $W^{s}(p)$ by $l(I)$. Define a u-rectangle to be an open set $S$ contained in $W^{u}(p)$, whose closure is an imbedded rectangle bounded by $W^{u}\left(\theta_{0}\right), W^{u u}(p)$ and two segments of $W^{s}(p)$. The union of the segments of $W^{u}\left(\theta_{0}\right)$ and $W^{u u}(p)$ bounding $S$, is denoted by $\partial^{u} S$.

To prove that $H D^{s}(\Lambda)<1$ it is sufficient to find $\beta \in(0,1)$ and a sequence $\left(U_{n}\right)_{n}$ of coverings of $\Lambda \cap W_{\text {loc }}^{s}(p)$ such that diam $\mathscr{U}_{n} \rightarrow 0$ and $\left(m_{\beta}\left(\mathscr{U}_{n}\right)\right)_{n}$ is bounded. Let $A_{0}$ be a $u$-rectangle with $\left\{\theta_{0}, p\right\} \subset \partial^{u} A_{0}$ and $x \in W \cap W^{u}\left(\theta_{0}\right), y \in W \cap W^{u u}(p)$ be the two smallest (for the $<$ ordering) elements of $W \cap \partial^{u} A_{0}$. Let $G_{j}=\left(f^{j}(x), f^{j}(y)\right.$ ), $j \geq 0$, and $C_{j}=\left[f^{j}(y), f^{j-1}(x)\right], j \geq 1$, and take $\left(A_{j}\right)_{j \geq 0}$ to be a sequence of $u$-rectangles

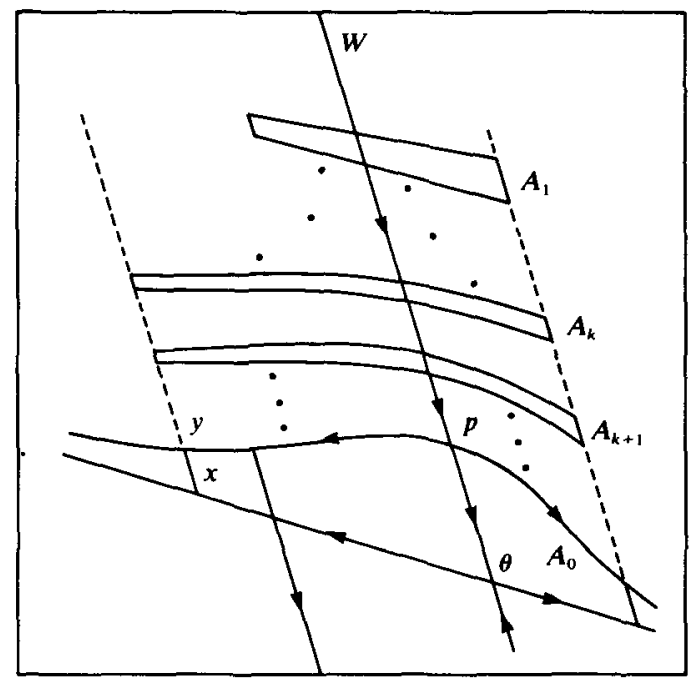

Figure 2 
such that

(P1) $A_{j} \cap[p, y]=G_{j}$

(P2) $f\left(A_{j}\right) \supset A_{j+1}$ for all $j \geq 0$ and

(P3) there is $k \geq 1$ such that for every connected component $C$ of $W-\left(\cup_{0}^{\infty} A_{j}\right)$ either $\partial C \subset\left(\partial^{u} A_{j-1} \cup \partial^{u} A_{j}\right), j \geq 2$, or $\partial C \subset\left(\partial^{u} A_{0} \cup \partial^{u} A_{j}\right)$ for some $j, 1 \leq j \leq k$ (see figure 2).

Replacing $f$ by $f^{k}$ we can, and do, assume $k=1$.

Let $\mathscr{C}$ be the family of connected components of $W-\left(\bigcup_{0}^{\infty} A_{j}\right)$ and, for $j \geq 1$, $\mathscr{C}_{j}=\left\{C \in \mathscr{C}: \partial C \subset \partial^{u} A_{j} \cup \partial^{u} A_{j-1}\right\}$. We define

$$
u_{n}=\left\{f^{n}(C) \cap[p, x]: C \in \mathscr{C}\right\}, \quad n \geq 0 .
$$

Note that $u_{n}$ is not really a covering of $\Lambda \cap[p, x]$ since it fails to cover some of the points in $W^{u u}(p) \cap[p, x]$ (more precisely: every point in $W^{u u}(p) \cap[p, x]$ is eventually not covered by $\left.\mathcal{U}_{n}\right)$. However this is unessential because $W^{u u}(p) \cap[p, x]$ is countable and Hausdorff dimensions of spaces differing by a countable set of points are equal.

Since diam $\mathcal{U}_{n} \rightarrow 0$ it is now enough to find $\beta \in(0,1)$ such that $m_{\beta}\left(\mathcal{U}_{0}\right)<\infty$ and $\left(m_{\beta}\left(\mathscr{U}_{n}\right)\right)_{n}$ is non-increasing. Let $U=f^{n-1}(C) \in \mathscr{U}_{n-1}, C \in \mathscr{C}$. If $C \in \mathscr{C}_{j}, j \geq 2$, then $f^{-1}(C) \in \mathscr{C}_{j-1}$. If $C \in \mathscr{C}_{1}$ then $f^{-1}(C)$ can be written

$$
\begin{aligned}
f^{-1}(C)= & \left(C_{1}^{1} \cup G_{1}^{1} \cup C_{2}^{1} \cup G_{2}^{1} \cup \cdots\right) \\
& \cup\left(G_{0}^{2} \cup C_{1}^{2} \cup G_{1}^{2} \cup C_{2}^{2} \cup G_{2}^{2} \cup \cdots\right) \cup \cdots \\
& \cup\left(G_{0}^{l} \cup C_{1}^{l} \cup G_{1}^{l} \cup C_{2}^{l} \cup G_{2}^{l} \cup \cdots\right),
\end{aligned}
$$

where the $G_{j}^{i}$ are connected components of $W \cap A_{j}$ and the $C_{j}^{i} \in \mathscr{C}_{j}$. Note that $l$ cannot be arbitrarily large because $l\left(f^{-1}(C)\right)$ is uniformly bounded above and the distance in $W$ between connected components of $W \cap A_{0}$ is uniformly bounded below. It follows that $U=f^{n}\left(f^{-1} C\right)$, either is also an element of $U_{n}$, or can be written

$$
U=\left(U_{1}^{1} \cup V_{1}^{1} \cup \cdots\right) \cup\left(V_{0}^{2} \cup U_{1}^{2} \cup V_{1}^{2} \cup \cdots\right) \cup \cdots \cup\left(V_{0}^{l} \cup U_{1}^{l} \cup V_{1}^{l} \cup \cdots\right),
$$

with $V_{j}^{i}=f^{n}\left(G_{j}^{i}\right), \quad U_{j}^{i}=f^{n}\left(C_{j}^{i}\right) \in \mathscr{U}_{n}$ and $l$ uniformly bounded. To get $m_{\beta}\left(U_{n}\right) \leq m_{\beta}\left(U_{n-1}\right)$ we should show that $\sum_{i, j}\left(l\left(U_{j}^{i}\right)\right)^{\beta} \leq(l(U))^{\beta}$ for all $U \in \mathcal{U}_{n-1}$ (and some suitable $\beta \in(0,1)$ independent of $U$ and $n$ ). This is done by taking $x_{j}^{i}=l\left(U_{j}^{i}\right) / l(U), y_{j}^{i}=l\left(V_{j}^{i}\right) / l(U)$ and $z_{j}=l\left(\left[f^{j}(x), f^{j-1}(x)\right]\right)$ in the following lemma.

LEMMA 3.1. Given $l \geq 1, a>0, b>0, \alpha \in(0,1)$ and $\left(z_{j}\right)_{j \geq 1}$ a sequence in $[0,1]$ with $\sum_{1}^{\infty} z_{j}^{\alpha}<\infty$, there is $\beta=\beta\left(l, a, b, \alpha,\left(z_{j}\right)_{j}\right) \in(\alpha, 1)$ such that for all sequences $\left(x_{j}^{i}\right)_{j \geq 1}$ and $\left(y_{j}^{i}\right)_{j \geq 1}, 1 \leq i \leq l$, in $[0,1]$ satisfying

(1) $\sum_{i, j}\left(x_{j}^{i}+y_{j}^{i}\right) \leq 1$

(2) $x_{j}^{i} \leq a y_{j}^{i}$ for all $j \geq 1$ and $1 \leq i \leq l$

(3) $\left(x_{j}^{i}+y_{j}^{i}\right) \leq b z_{j}$ for all $j \geq 1$ and $1 \leq i \leq l$ we have $\sum_{i, j}\left(x_{j}^{i}\right)^{\beta} \leq 1$.

We leave the proof of this result to the end of this section and proceed now to find $\alpha \in(0,1), a>0$ and $b>0$ such that the sequences $\left(z_{j}\right)_{j},\left(x_{j}^{i}\right)_{j}$ and $\left(y_{j}^{i}\right)_{j}$ above satisfy the conditions in Lemma 3.1.

LEMMA 3.2. $\sum_{1}^{\infty} l\left(\left[f^{j}(x), f^{j-1}(x)\right]\right)^{a}$ converges, for all $\alpha>\frac{1}{2}$. 
Proof. Let $\varphi:[0,+\infty) \rightarrow W^{s}(p)$ be the parametrization of $W^{s}(p)$ by arc-length and $\tilde{f}=\varphi^{-1} \circ\left(\left.f\right|_{W^{s}(p)}\right) \circ \varphi:[0,+\infty) \rightarrow[0,+\infty)$ and denote $\tilde{x}_{j}=\varphi^{-1}\left(f^{j}(x)\right)$. Since $p$ is a quadratic saddle-node for $f$ there are $t_{0}>0$ and $a_{1}>0$ such that $t-2 a_{1} t^{2} \leq \tilde{f}(t) \leq t-a_{1} t^{2}$ for all $0 \leq t \leq t_{0}$. Take $m \geq 1$ such that $\tilde{x}_{m} \leq t_{0}$ and $m a_{1} t_{0} \geq 1$ and let $a_{2}=\max \left\{m \cdot \tilde{x}_{m}, a_{1}^{-1}\right\}$. By induction we have $\tilde{x}_{j} \leq a_{2} / j$ for all $j \geq m$ :

$$
\begin{aligned}
& \tilde{x}_{j} \leq a_{2} / j \leq a_{2} / m \leq t_{0} \Rightarrow \tilde{x}_{j+1}=\tilde{f}\left(\tilde{x}_{j}\right) \leq \tilde{f}\left(a_{2} / j\right) \leq a_{2} / j-a_{1} a_{2}^{2} / j^{2} \\
& =\left(a_{2} /(j+1)\right) \cdot\left[(j+1) \cdot\left(j-a_{1} a_{2}\right) / j^{2}\right] \leq a_{2} /(j+1) .
\end{aligned}
$$

It follows that, for $j>m$,

$$
l\left(\left[f^{j}(x), f^{j-1}(x)\right]\right)=\left(\tilde{x}_{j-1}-\tilde{f}\left(\tilde{x}_{j-1}\right)\right) \leq 2 a_{1}\left(\tilde{x}_{j-1}\right)^{2} \leq 2 a_{1} a_{2}^{2} /(j-1)^{2}
$$

and this clearly implies the lemma.

Observe that

$$
m_{\beta}\left(U_{0}\right)=\sum_{1}^{\infty} l\left(\left[f^{j}(y), f^{j-1}(x)\right]\right)^{\beta} \leq \sum_{1}^{\infty} l\left(\left[f^{j}(x), f^{j-1}(x)\right]\right)^{\beta},
$$

so Lemma 3.2 already implies that $m_{\beta}\left(\mathcal{U}_{0}\right)<\infty$ for all $\beta \in(1 / 2,1)$.

The next result is the main step in the proof of Theorem $A$. We postpone its proof to $\$ 4$.

Lemma 3.3. (Distortion Lemma). There is $c>0$ such that $\sum_{0}^{\infty} l\left(f^{j}(C \cup G)\right) \leq c$ for all connected component $C$ of $W-\left(\bigcup_{0}^{\infty} A_{j}\right)$ and all connected components $G$ of $W \cap\left(\bigcup_{0}^{\infty} A_{j}\right)$ with $\partial C \cap \partial G \neq \phi$.

The sequences $\left(x_{j}^{i}=l\left(U_{j}^{i}\right) / l(U)\right)_{j}$ and $\left(y_{j}^{i}=l\left(V_{j}^{i}\right) / l(U)\right)_{j}$ that we are considering, clearly, satisfy condition (1) in Lemma 3.1 , so we are left to find $a>0$ and $b>0$ as in (2) and (3). This is done in the following corollaries of the Distortion Lemma.

COROLlary 3.4. There is $a>0$ such that for all $C$ and $G$ as in Lemma 3.3 we have $a^{-1} \leq l\left(f^{n}(C)\right) / l\left(f^{n}(G)\right) \leq a$ for all $n \geq 0$.

Proof. Every $C \in \mathscr{C}$ can be written as $C=f^{j}\left(C_{0}\right)$ for some $j \geq 0$ and $C_{0} \in \mathscr{C}_{1}$, so we can assume from the beginning that $C \in \mathscr{C}_{1}$ andf $G$ is a connected component of $W \cap A_{0}$ or $W \cap A_{1}$. We note that the set of values of $(l(C) / l(G))$ for such $C$ and $G$ is bounded away from 0 and $+\infty$. Now, for $n \geq 0$,

$$
\left[l\left(f^{n}(C)\right) / l\left(f^{n}(G)\right)\right]=[l(C) / l(G)] \cdot\left[\left|D^{s} f^{n}(\xi)\right| /\left|D^{s} f^{n}(\eta)\right|\right]
$$

for some $\xi \in C, \eta \in G$. This can be written

$$
\begin{aligned}
{\left[l\left(f^{n}(C)\right) / l\left(f^{n}(G)\right)\right] } & =[l(C) / l(G)] \exp \left(\sum_{0}^{n-1}\left(\log \left|D^{s} f\right|\left(f^{j} \xi\right)-\log \left|D^{s} f\right|\left(f^{j} \eta\right)\right)\right) \\
& \leq[l(C) / l(G)] \exp \left(\operatorname{Lip}\left(\log \left|D^{s} f\right|\right) \cdot \sum_{0}^{n-1} l\left(f^{j}(C \cup G)\right)\right) \\
& \leq[l(C) / l(G)] \exp \left(c \operatorname{Lip}\left(\log \left|D^{s} f\right|\right)\right) \\
& \leq a
\end{aligned}
$$

for some universal $a>0$. The other inequality is obtained in the same way and this completes the proof of the corollary. 
Corollary 3.5. There is $b>0$ such that for $C \in \mathscr{C}_{1}$

$$
b^{-1} l\left(\left[f^{j}(x), f^{j-1}(x)\right]\right) \leq\left[l\left(f^{n}\left(C_{j} \cup C_{j}\right)\right) / l\left(f^{n-1}(C)\right)\right] \leq b \cdot l\left(\left[f^{j}(x), f^{j-1}(x)\right]\right),
$$

for all $C_{j} \in \mathscr{C}_{j}$ and $G_{j}$ a connected component of $W \cap A_{j}$, with $\left(C_{j} \cup G_{j}\right) \subset f^{-1}(C)$, and all $n>0$.

Proof. As in the proof of Corollary 3.4 we get

$$
\begin{aligned}
& {\left[l\left(f^{n}\left(C_{j} \cup G_{j}\right)\right) / l\left(f^{n-1}(C)\right)\right]} \\
& \quad \leq\left[l\left(C_{j} \cup G_{j}\right) / l\left(f^{-1} C\right)\right] \cdot \exp \left(\operatorname{Lip}\left(\log \left|D^{s} f\right|\right) \sum_{0}^{n-1} l\left(f^{-1}(C)\right)\right) .
\end{aligned}
$$

Note that $l\left(C_{j} \cup G_{j}\right) \leq r \cdot l\left(\left[f^{j}(x), f^{j-1}(x)\right]\right)$, where $r>0$ is a Lipschitz constant for the unstable holonomy projection $\pi^{\text {u }}($ see $\S 2)$. Then

$$
\left[l\left(f^{n}\left(C_{j} \cup G_{j}\right)\right) / l\left(f^{n-1}(C)\right)\right] \leq r b_{1} \exp \left(\operatorname{Lip}\left(\log \left|D^{s} f\right|\right) \cdot\left(b_{1}+c\right)\right)
$$

with $b_{1}>0$ such that $b_{1}^{-1} \leq l\left(f^{-1}(C)\right) \leq b_{1}$, for all $C \in \mathscr{C}_{1}$. The other inequality follows in the same way. The proof of the corollary is complete.

We end this section by proving Lemma 3.1 .

Proof of Lemma 3.1. Since

and

$$
\sum_{i, j}\left(x_{j}^{i}\right)^{\beta} \leq\left(1+a^{-1}\right)^{-\beta} \sum_{i, j}\left(x_{j}^{i}+y_{j}^{i}\right)^{\beta}
$$

$$
\left(1+a^{-1}\right)^{-\beta} \rightarrow\left(1+a^{-1}\right)^{-1}<1 \quad \text { when } \beta \rightarrow 1,
$$

it is sufficient to show that $\sum_{i, j}\left(x_{j}^{i}+y_{j}^{i}\right)^{\beta}$ is close to $\sum_{i, j}\left(x_{j}^{i}+y_{j}^{i}\right) \leq 1$, for $\beta$ close to 1. Now, for $\alpha<\beta<1$,

$$
\begin{aligned}
\sum_{i, j}\left[\left(x_{j}^{i}+y_{j}^{i}\right)^{\beta}-\left(x_{j}^{i}+y_{j}^{i}\right)\right] & \leq \sum_{\substack{1 \leq i \leq 1 \\
1 \leq j \leq n}}\left[\left(x_{j}^{i}+y_{j}^{i}\right)^{\beta}-\left(x_{j}^{i}+y_{j}^{i}\right)\right]+\sum_{\substack{1 \leq i \leq l \\
j>n}}\left(x_{j}^{i}+y_{j}^{i}\right)^{\alpha} \\
& \leq \sum_{\substack{l \leq i \leq 1 \\
l \leq j \leq n}}\left[\left(x_{j}^{i}+y_{j}^{i}\right)^{\beta}-\left(x_{j}^{i}+y_{j}^{i}\right)\right]+l \cdot b^{\alpha} \cdot \sum_{j>n} z_{j}^{\alpha}
\end{aligned}
$$

Let $\delta>0$ and take $n \geq 1$ such that $\sum_{j>n} z_{j}^{\alpha} \leq \delta / 2 l b^{\alpha}$ and $\beta$ close to 1 so that $\left(\xi^{\beta}-\xi\right) \leq \delta / 2 \ln$ for all $\xi \in[0,1]$. Then

$$
\sum_{i, j}\left[\left(x_{j}^{i}+y_{j}^{i}\right)^{\beta}-\left(x_{j}^{i}+y_{j}^{i}\right)\right] \leq \ln (\delta / 2 \ln )+l b^{\alpha}\left(\delta / 2 l b^{\alpha}\right)=\delta .
$$

This proves the lemma.

\section{Proof of the distortion lemma}

For the sake of clearness we divide the proof into three steps:

Step 1. Let $C$ and $G$ be as in the statement of the lemma. Clearly $\left(l\left(f^{j}(C \cup G)\right)\right)_{j \geq 0}$ is decreasing and $l\left(f^{j+1}(C \cup G)\right) \leq \lambda_{0} l\left(f^{j}(C \cup G)\right)$ whenever $f^{j}(C \cup G) \cap Q=\phi$. Moreover $\left(f^{j}(C \cup G)\right)_{j \geq 0}$ is a disjoint family. To prove this we only need to show that $\sup _{w}(f(C \cup G)) \leq \inf _{W}(C \cup G)$, since $\left.f\right|_{w}: W \rightarrow W$ is a homeomorphism with $f(x)<x$ for all $x \in W$. Let $G=(\alpha, \beta)$ and $C=[\beta, \gamma]$ with $\alpha<\beta<\gamma$. Then:

(i) $f(\alpha)<\alpha$,

(ii) $f(\beta)<\alpha(f(\beta) \neq \alpha, f(\beta)<\beta$ and $f(\beta) \notin(\alpha, \beta))$, and 
(iii) $f(\gamma) \leq \alpha\left(f(\gamma)>\alpha\right.$ implies $f^{-1}(\alpha) \in(\beta, \gamma)$ and this is absurd since $\left.f^{-1}\left(\bigcup_{0}^{\infty} \partial^{u} A_{j}\right) \subset\left(\bigcup_{0}^{\infty} \partial^{u} A_{j}\right)\right)$.

Case $\alpha>\beta>\gamma$ is analogous.

Step 2. Let $G_{j}=\left(f^{j}(x), f^{j}(y)\right), j \geq 0$, and $C_{j}=\left[f^{j}(y), f^{j-1}(x)\right], j \geq 1$. Clearly $l\left(G_{0}\right)+\sum_{1}^{\infty} l\left(C_{j} \cup G_{j}\right)=l([p, y])$. We claim that there is $a_{1}>0$ such that, for all $I, J$ intervals in $W$ contained in $\left(C_{k} \cup G_{k}\right.$ ) (resp. $G_{k-1} \cup C_{k}$ ) for some $k \geq 1$, we have

$$
a_{1}^{-1}[l(I) / l(J)] \leq\left[l\left(f^{n}(I)\right) / l\left(f^{n}(J)\right)\right] \leq a_{1}[l(I) / l(J)]
$$

for all $n \geq 0$. To prove this we write:

$$
\begin{aligned}
{\left[l\left(f^{n}(I)\right) / l\left(f^{n}(J)\right)\right] } & =[l(I) / l(J)] \cdot\left[\left|D^{s} f^{n}(\xi)\right| /\left|D^{s} f^{n}(\eta)\right|\right] \\
& =[l(I) / l(J)] \exp \left(\sum_{0}^{n-1}\left(\log \left|D^{s} f\right|\left(f^{i} \xi\right)-\log \left|D^{s} f\right|\left(f^{i} \eta\right)\right)\right) \\
& \leq[l(I) / l(J)] \cdot \exp \left(\operatorname{Lip}\left(\log \left|D^{s} f\right|\right) \sum_{0}^{n-1} d\left(f^{i} \xi, f^{i} \eta\right)\right) \\
& \leq[l(I) / l(J)] \cdot \exp \left(\operatorname{Lip}\left(\log \left|D^{s} f\right|\right) \cdot l([p, y])\right),
\end{aligned}
$$

where $\xi \in I$ and $\eta \in J$. The other inequality is obtained in the same way.

Step 3. Take $0=m_{0} \leq n_{0}<m_{1}<n_{1}<\cdots<m_{s}<n_{s}<m_{s+1}=\infty$ such that

(i) $f^{j}(C \cup G) \cap Q=\phi$ for $m_{i} \leq j<n_{i}$ and

(ii) $f^{j}(C \cup G) \cap Q \neq \phi$ for $n_{i} \leq j<m_{i+1}, 0 \leq i \leq s$,

and write

$$
\sum_{0}^{\infty} l\left(f^{j}(C \cup G)\right)=\sum_{i=0}^{s}\left(\sum_{j=m_{i}}^{n_{i}-1} l\left(f^{j}(C \cup G)\right)+\sum_{j=n_{i}}^{m_{i+1}-1} l\left(f^{j}(C \cup G)\right)\right) .
$$

Note that, by Step 1 ,

$$
l\left(f^{n_{i}}(C \cup G)\right) \leq \lambda_{0}^{n_{i}-m_{i}} l\left(f^{m_{i}}(C \cup G)\right) \leq \lambda_{0}\left(f^{m_{i}}(C \cup G)\right)
$$

at least for $i>0$, and

$$
l\left(f^{m_{i+1}}(C \cup G)\right) \leq l\left(f^{n_{i}}(C \cup G)\right)
$$

for all $i \geq 0$. Therefore

and, by induction,

$$
l\left(f^{m_{i+1}}(C \cup G)\right) \leq \lambda_{0} l\left(f^{m_{i}}(C \cup G)\right)
$$

$$
l\left(f^{m_{i+1}}(C \cup G)\right) \leq \lambda_{0}^{i} l(C \cup G)
$$

for all $i \geq 0$. Hence

$$
\begin{aligned}
\sum_{i=0}^{s} \sum_{j=m_{i}}^{n_{i-1}} l\left(f^{j}(C \cup G)\right) & \leq \sum_{i=0}^{s} \sum_{j=m_{i}}^{n_{i}-1} \lambda_{0}^{j-m_{i}} \cdot l\left(f^{m_{i}}(C \cup G)\right) \\
& \leq \sum_{i=0}^{s} \sum_{j=m_{i}}^{n_{i}-1} \lambda_{0}^{j-m_{i}} \cdot \lambda_{0}^{i-1} l(C \cup G) \leq \lambda_{0}^{-1}\left(\sum_{0}^{\infty} \lambda_{0}^{i}\right)^{2} a_{2},
\end{aligned}
$$

where $a_{2}$ is a uniform upper bound for $l(C \cup G)$, with $C$ and $G$ as in the statement of the lemma.

Now we estimate $\sum_{j=n_{i}}^{m_{i+1}-1} l\left(f^{j}(C \cup G)\right)$. Consider first $i=0$ and define for $l \geq 0$,

$$
H_{i}^{0}=f^{l}\left(\pi^{u}\left(f^{n_{0}}(C \cup G)\right)\right)
$$


where $\pi^{u}: Q \rightarrow[p, y]$ is the projection along the leaves of $\mathscr{F}^{u}$ in $Q$. Then, by Step $1,\left(H_{l}^{0}\right)_{l \geq 0}$ is disjoint, so

$$
\sum_{j=n_{0}}^{m_{1}-1} l\left(f^{j}(C \cup G)\right) \leq r \sum_{l \geq 0} l\left(H_{l}^{0}\right) \leq r \cdot l([p, y]),
$$

where $r$ is a Lipschitz constant for $\pi^{u}$ (see figure 3). Define now, for $i \geq 1, l \geq 0$,

$$
H_{i}^{i}=f^{l}\left(\pi^{u}\left(f^{n_{i}}(C \cup G)\right)\right) \text {. }
$$

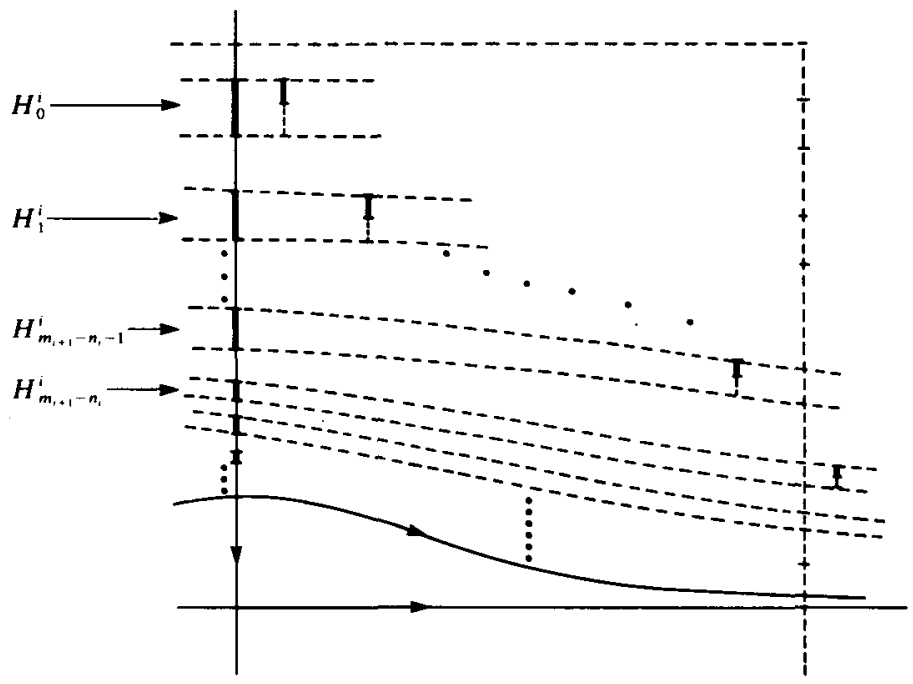

FIGURE 3

Since both $\left(H_{l}^{0}\right)_{l}$ and $\left(H_{l}^{i}\right)_{l}$ are disjoint families we must have $\left(H_{0}^{0} \cup H_{0}^{i}\right)$ contained in some fundamental domain $\left(C_{k} \cup G_{k}\right)$ (or $\left.G_{k-1} \cup C_{k}\right)$ of $W$. Therefore, by Step 2,

$$
\begin{aligned}
l\left(H_{l}^{i}\right) \leq & a_{1} l\left(H_{l}^{0}\right)\left[l\left(H_{0}^{i}\right) / l\left(H_{0}^{0}\right)\right] \\
\leq & a_{1} l\left(H_{l}^{0}\right)\left[l\left(H_{0}^{i}\right) / l\left(f^{n_{i}}(C \cup G)\right)\right]\left[l\left(f^{n_{i}}(C \cup G)\right) / l\left(f^{n_{0}}(C \cup G)\right)\right] \\
& \times\left[l\left(f^{n_{0}}(C \cup G)\right) / l\left(H_{0}^{0}\right)\right] \\
\leq & a_{1} r \lambda_{0}^{i} r l\left(H_{l}^{0}\right)
\end{aligned}
$$

for all $l \geq 0$. It follows that

$$
\begin{aligned}
\sum_{j=n_{i}}^{m_{i+1}-1} l\left(f^{j}(C \cup G)\right) & \leq r \sum_{l \geq 0} l\left(H_{l}^{i}\right) \\
& \leq a_{1} r^{3} \lambda_{0}^{i} \sum_{l \geq 0} l\left(H_{l}^{0}\right) \leq a_{1} r^{3} \lambda_{0}^{i} l([p, y]) .
\end{aligned}
$$

Finally we get

$$
\begin{aligned}
\sum_{i=0}^{s} \sum_{j=n_{i}}^{m_{i+1}-1} l\left(f^{j}(C \cup G)\right) & \leq \sum_{i=0}^{s} a_{1} r^{3} \lambda_{0}^{i} l([p, y]) \\
& \leq a_{1} r^{3} l([p, y])\left(\sum_{0}^{\infty} \lambda_{0}^{i}\right) .
\end{aligned}
$$

The lemma now follows from (1)-(3). 
5. $H D^{s}\left(\Lambda_{0}\right)=d^{s}\left(\Lambda_{0}\right)>\frac{1}{2}$

In order to prove that the Hausdorff dimension and the limit capacity of $\Lambda \cap[p, x]$ are equal we need to refine the analysis of $\S 3$. Our argument uses ideas from the proof of the corresponding result in the hyperbolic case, see Takens [11].

We begin by noting that for every $C \in \mathscr{C}_{1}$ there are:

(i) intervals $H_{1}, \ldots, H_{k}$ covering $f^{-1}(C)$, and

(ii) for each $1 \leq i \leq k, \pi_{i}^{u}: H_{i} \rightarrow[p, x]$ an $\mathscr{F}^{u}$-holonomy which is an $r$-Lipschitz homeomorphism with $r$-Lipschitz inverse (see figure 4).

Moreover $k$ and $r$ may be taken independent of $C \in \mathscr{C}_{1}$.

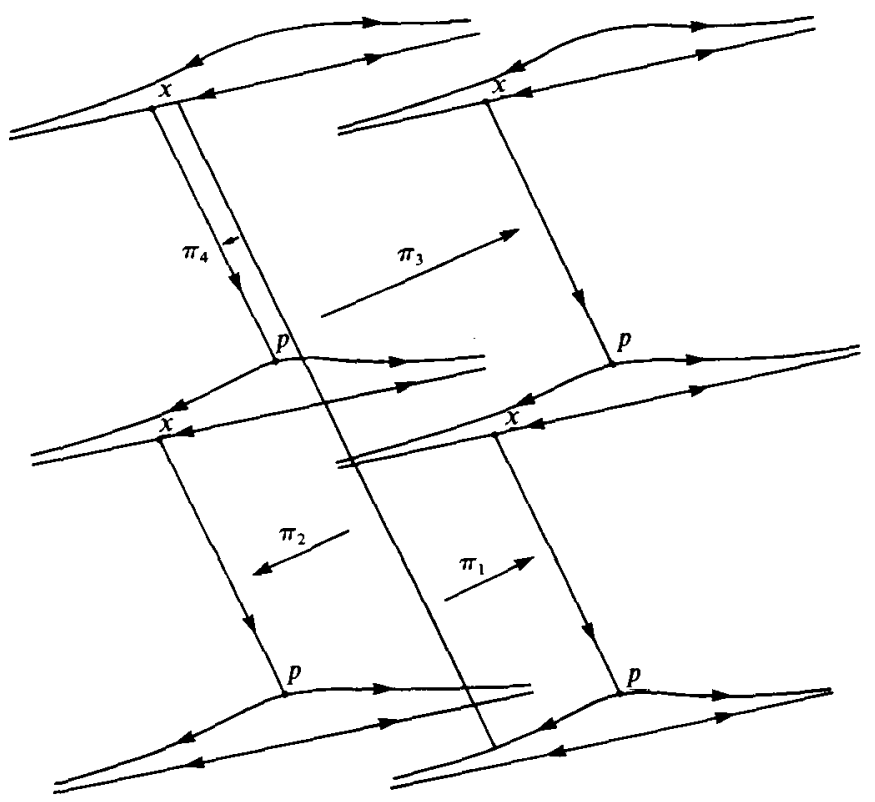

Figure 4

We now construct a sequence $\left(\mathscr{K}^{n}\right)_{n \geqslant 1}$ of coverings of $\Lambda \cap[p, x]$. These will be used to give estimates of $H D(\Lambda \cap[p, x])$ and $d(\Lambda \cap[p, x])$ which imply their equality. Define

$$
\mathscr{K}^{1}=\left\{K_{j}: j \geq 1\right\},
$$

where $K_{j}=\left[f^{j}(y), f^{j-1}(x)\right]$. Note that, for all $j \geq 1$,

$$
f^{-j+1}\left(K_{j}\right)=K_{1} \in \mathscr{C}_{1} \text {. }
$$

Therefore (recall §3)

$$
\begin{aligned}
f^{-j}\left(K_{j}\right) & =\left(C_{j, 1}^{1} \cup G_{j, 1}^{1} \cup C_{j, 2}^{1} \cup \cdots\right) \cup G_{j, 0}^{2} \\
& \cup\left(C_{j, 1}^{2} \cup G_{j, 1}^{2} \cup C_{j, 2}^{2} \cup \cdots\right) \cup \cdots \cup G_{j, 0}^{l} \\
& \cup\left(C_{j, 1}^{l} \cup G_{j, 1}^{l} \cup C_{j, 2}^{l} \cup \cdots\right)
\end{aligned}
$$

with $C_{j, K}^{s} \in \mathscr{C}_{k}, G_{j, k}^{s}$ a connected component of $W \cap A_{k}$ and $l \geq 1$ uniformly bounded 
above by some $l_{0} \geq 1$. Let $K_{j, k}^{s}=f^{j}\left(C_{j, k}^{s}\right) \subset K_{j}$ and define

$$
\mathscr{K}^{2}=\left\{K_{j, k}^{s}: j \geq 1, k \geq 1 \text { and } 1 \leq s \leq l(j) \leq l_{0}\right\} .
$$

Note that for all $K_{j, k}^{s} \in \mathscr{K}^{2}$ we have

$$
f^{(j+k)+1}\left(K_{j, k}^{s}\right) \in \mathscr{C}_{1} .
$$

Suppose now already constructed

$$
\mathscr{K}^{n}=\left\{K_{j_{1}, \ldots, j_{n}}^{s}: j_{1} \geq 1, \ldots, j_{n} \geq 1 \quad \text { and } \quad 1 \leq s \leq l\left(j_{1}, \ldots, j_{n-1}\right) \leq l_{0}^{n-1}\right\}
$$

with

$$
f^{-\left(j_{1}+\cdots+j_{n}\right)+1}\left(K_{j_{1}, \ldots, j_{n}}^{s}\right) \in \mathscr{C}_{1}
$$

for all $K_{j_{1}, \ldots, j_{n}}^{s} \in \mathscr{K}^{n}$. Then

$$
\begin{aligned}
f^{-\left(j_{1}+\cdots+j_{n}\right)}\left(K_{j_{1}, \ldots, j_{n}}^{s}\right) & =\left(C_{j_{1}, \ldots, j_{n, 1}}^{s, 1} \cup G_{j_{1}, \ldots, j_{n, 1}}^{s, 1} \cup C_{j_{1}, \ldots, j_{n, 2}}^{s, 1} \cup \cdots\right) \cup \cdots \cup G_{j_{1}, \ldots, j_{n, 0}}^{s, t} \\
& \cup\left(C_{j_{1}, \ldots, j_{n, 1}}^{s, t} \cup G_{j_{1}, \ldots, j_{n, 1}}^{s, t} \cup C_{j_{1}, \ldots, j_{n, 2}}^{s, t} \cup \cdots\right)
\end{aligned}
$$

with each $C_{j_{1}, \ldots, j_{n}, j_{n+1}}^{s, t} \in \mathscr{C}_{j_{n+1}}$. We define $\mathscr{K}^{n+1}$ as the family of

$$
K_{j_{1}, \ldots, j_{n}, j_{n+1}}^{s, t}=f^{\left(j_{1}+\cdots+j_{n}\right)}\left(C_{j_{1}, \ldots, j_{n}, j_{n+1}}^{s, t}\right) \subset K_{j_{1}, \ldots, j_{n}}^{s}
$$

obtained in this way. Clearly it can be written as

$$
\mathscr{K}^{n+1}=\left\{K_{j_{1}, \ldots, j_{n}, j_{n+1}}^{s}: j_{1} \geq 1, \ldots, j_{n+1} \geq 1 \text { and } 1 \leq s \leq l\left(j_{1}, \ldots, j_{n}\right) \leq l_{0}^{n}\right\} .
$$

For the sake of notational simplicity we will, from now on, write $J$ and $|J|$ in place of $j_{1}, \ldots, j_{n}$, and $j_{1}+\cdots+j_{n}$ respectively. Define, for $K_{J}^{s} \in \mathscr{K}^{n}$,

$$
\lambda_{J}^{s}=\inf \mid D^{s} f^{|J|}{\mid \tilde{K}_{j}^{s}}_{\mid}
$$

and

$$
\Lambda_{J}^{s}=\sup \left|D^{s} f^{|J|} \tilde{K}_{J}^{s}\right|
$$

where $\tilde{K}_{J}^{s}=f^{|J|}\left(K_{J}^{s}\right)$. Let, for $\gamma>0$ and $n \geq 1$,

$$
\lambda_{n}(\gamma)=\sum_{s, J}\left(\lambda_{J}^{s} / r\right)^{\gamma}
$$

and

$$
\Lambda_{n}(\gamma)=k \sum_{s, J}\left(r \Lambda_{J}^{s}\right)^{\gamma},
$$

where $k \geq 1$ and $r>0$ are as above.

\section{Proposition 5.1.}

(a) For $n \geq 1$ big enough, $\lambda_{n}$ and $\Lambda_{n}$ are finite, continuous and strictly decreasing functions in $\left(\frac{1}{2},+\infty\right)$. Moreover $\lim _{\gamma \rightarrow+\infty} \lambda_{n}(\gamma)=\lim _{\gamma \rightarrow+\infty} \Lambda_{n}(\gamma)=0$ and $\lim _{\gamma \rightarrow(1 / 2)+} \lambda_{n}(\gamma)=\lim _{\gamma \rightarrow(1 / 2)+} \Lambda_{n}(\gamma)+\infty$.

(b) Let, for $n \geq 1$ big, $\alpha_{n}, \beta_{n} \in\left(\frac{1}{2},+\infty\right)$ be such that $\lambda_{n}\left(\alpha_{n}\right)=1=\Lambda_{n}\left(\beta_{n}\right)$. Then:

(i) $H D(\Lambda \cap[p, x]) \geq \alpha_{n}$,

(ii) $d(\Lambda \cap[p, x]) \leq \beta_{n}$,

(iii) $\left(\beta_{n}-\alpha_{n}\right)_{n} \rightarrow 0$. 
Clearly Proposition 5.1 implies Theorem $A^{\prime}(\mathrm{c})$. We collect in the following lemma the main estimates used in the proof of this proposition.

Denote by $p_{J}^{s}$ the accumulation point of the sequence $\left(K_{J, j}^{s}\right)_{j}$ and let $\Delta_{J, j}^{s}$ be the length of the convex hull (in $W$ ) of $K_{J, j}^{s} \cup\left\{p_{J}^{s}\right\}$.

LEMMA 5.2. There are $a, b, d, e>0$ and $0<\lambda_{0}<1$ such that

(a) $a^{-1} \lambda_{J}^{s} \leq l\left(K_{J}^{s}\right) \leq a \Lambda_{J}^{s}$,

(b) $\Lambda_{J}^{s} \leq b \lambda_{J}^{s}$,

(c) $\Lambda_{j}^{s} \leq \lambda_{0}^{n-1}$,

(d) $\prod_{1}^{n} d^{-1} / j_{i}^{2} \leq l\left(K_{J}^{s}\right) \leq \prod_{1}^{n} d / j_{i}^{2}$,

(e) $\Delta_{,, j}^{s} \leq(e / j) \prod_{1}^{n} d / j_{i}^{2}$,

for all $n, J=\left(j_{1}, \ldots, j_{n}\right), j$ and $s$.

Proof.

(a) Just take $a>0$ such that $a^{-1} \leq l\left(f^{-1}(C)\right) \leq a$ for all $C \in \mathscr{C}_{1}$ and note that $l\left(K_{J}^{s}\right)=l\left(\tilde{K}_{J}^{s}\right) \cdot\left|D^{s} f^{|J|}(\xi)\right|$ for some $\xi \in \tilde{K}_{J}$.

(b) From the definitions

$$
\Lambda_{J}^{s} / \lambda_{J}^{s} \leq \exp \left(\operatorname{Lip}\left(\log \left|D^{s} f\right|\right) \cdot \sum_{0}^{|J|-1} l\left(f^{j}\left(\tilde{K}_{J}^{s}\right)\right)\right) \leq \exp \left(\operatorname{Lip}\left(\log \left|D^{s} f\right|\right) \cdot(a+c)\right)
$$

where $c>0$ is given by Lemma 3.3 and $a>0$ is as in (a).

(c) Take $\lambda_{0}=\sup \left\{\left|D^{s} f(\xi)\right|: \xi \in C\right.$ and $\left.C \in \mathscr{C}_{1}\right\}$. Noting that for all $1 \leq i \leq n$, $f^{\left(j_{1}+\cdots+j_{i-1}\right)+1}\left(\tilde{K}_{J}^{s}\right)$ is contained in some $C_{i}^{\prime} \in \mathscr{C}_{1}$, we get

$$
\Lambda_{J}^{s} \leq \prod_{1}^{n-1} \sup \left|D^{s} f\right|_{C_{i}^{\prime}} \mid \leq \lambda_{0}^{n-1} \text {. }
$$

(d) We first prove the lower bound for $n=1$. Let $\varphi, \tilde{f}, \tilde{x}_{j}, t_{0}, a_{1}$ and $m$ be as in Lemma 3.2. Take $a_{3}>0$ such that $a_{3} \leq \min \left\{m \tilde{x}_{m}, a_{1} / 4\right\}$. Since

$$
a_{3} / j \leq \tilde{x}_{j} \leq t_{0} \Rightarrow \tilde{x}_{j+1} \geq \tilde{f}\left(a_{3} / j\right) \geq a_{3} / j-2\left(a_{1} a_{3}^{2} / j^{2}\right) \geq a_{3} /(j+1)
$$

we have $\tilde{x}_{j} \geq a_{3} / j$ for all $j \geq m$. Then, for $j>m$

$$
\left(\tilde{x}_{j-1}-\tilde{x}_{j}\right) \geq a_{1}\left(\tilde{x}_{j-1}\right)^{2} \geq a_{1} a_{3}^{2} /(j-1)^{2}
$$

and this, as $\left(l\left(K_{j}\right) / l\left(\left[f^{j}(x), f^{j-1}(x)\right]\right)\right)$ is bounded away from zero (see Step 2 in $\S 4)$, implies $l\left(K_{j}\right) \geq d_{1} / j^{2}$, for some $d_{1}>0$ and all $j \geq 1$.

Now for any $K_{J}^{s} \in \mathscr{K}^{n}$ take $K_{I}^{t} \in \mathscr{K}^{n-1}, I=\left(j_{1}, \ldots, j_{n-1}\right)$, such that $K_{J}^{s} \subset K_{I}^{t}$. Then

$$
\left[l\left(K_{J}^{s}\right) / l\left(K_{I}^{t}\right)\right]=\left[D^{s} f^{|I|}(\xi) / D^{s} f^{I I \mid}(\eta)\right]\left[l\left(f^{-|I|}\left(K_{J}^{s}\right)\right) / l\left(f^{-|I|}\left(K_{I}^{t}\right)\right)\right]
$$

for some $\xi, \eta$ in $f^{-|I|}\left(K_{I}^{t}\right)$. By construction of $K_{J}^{s}$ we have $f^{-|I|}\left(K_{J}^{s}\right) \in \mathscr{C}_{j_{n}}$, so $l\left(f^{-|I|} K_{J}^{s}\right) \geq r^{-1} l\left(K_{j_{n}}\right)$, with $r>0$ a Lipschitz constant for the unstable foliation holonomy. Also $f^{-|I|}\left(K_{I}^{t}\right) \in f^{-1}\left(\mathscr{C}_{1}\right)$ so $l\left(f^{-|I|}\left(K_{I}^{t}\right)\right) \leq a$. Replacing above we get

$$
l\left(K_{J}^{s}\right) \geq \exp \left(-\operatorname{Lip}\left(\log \left|D^{s} f\right|\right)(a+c)\right) \cdot r^{-1} a^{-1} l\left(K_{j_{n}}\right) l\left(K_{I}^{t}\right)
$$

By induction it follows

$$
l\left(K_{J}^{s}\right) \geq \prod_{1}^{n} \frac{d^{-1}}{j_{i}^{2}}
$$


if

$$
d \geq \exp \left(\operatorname{Lip}\left(\log \left|D^{s} f\right|\right) \cdot(a+c)\right) \operatorname{rad}_{1}^{-1} .
$$

For the upper bound, case $n=1$ is contained in the proof of Lemma 3.2 and the inductive step is proved as above.

(e) Case $n=0$ is contained in the proof of Lemma 3.2 and for the general case we show as in (d) that

$$
\Delta_{J, j}^{s} \leq r . a \cdot \exp \left(\operatorname{Lip}\left(\log \left|D^{s} f\right|\right)(a+c)\right) \Delta_{j} l\left(K_{J}^{s}\right) .
$$

This completes the proof of the lemma.

Proof of 5.1.a. From Lemma 5.2 we get in a straightforward way

$$
\Lambda_{n}(\gamma) \leq k l_{0}^{n}\left(r a b d^{n}\right)^{\gamma} \sum_{1}^{\infty}\left(j^{-2 \gamma}\right)^{n}
$$

Hence $\lambda_{n}(\gamma) \leq \Lambda_{n}(\gamma)<\infty$ for $\gamma>\frac{1}{2}$ and $\lambda_{n}, \Lambda_{n}$ are continuous on $\gamma$. It follows from Lemma $5.2(\mathrm{c})$ that $\sup _{s, J}\left\{\lambda_{J}^{s} / r, r \Lambda_{J}^{s}\right\}<1$ for $n \geq 1$ big. This implies that $\lambda_{n}$ and $\Lambda_{n}$ are decreasing and $\lim _{\gamma \rightarrow+\infty} \lambda_{n}(\gamma)=\lim _{\gamma \rightarrow+\infty} \Lambda_{n}(\gamma)=0$. Finally, from

$$
\lambda_{n}(\gamma) \geq\left(r a b d^{n}\right)^{-\gamma} \sum_{1}^{\infty}\left(j^{-2}\right)^{n}
$$

it follows $\lim _{\gamma \rightarrow(1 / 2)+} \lambda_{n}(\gamma)=\lim _{\gamma \rightarrow(1 / 2)+} \Lambda_{n}(\gamma)=+\infty$. This ends the proof of 5.1.a.

Proof of 5.1.b(i). Suppose $H D(\Lambda \cap[p, x])<\alpha<\alpha_{n}$. Then

$$
\sum\left(\lambda_{J}^{s} / r\right)^{\alpha}>1 \text {. }
$$

Take $\mathscr{J}$ a finite set of indices $(s, J)$ such that this inequality remains true when the sum is taken over $\mathscr{I}$. Let $\varepsilon_{0}$ be such that if $\mathscr{U}$ is a covering of $\Lambda \cap[p, x]$ with $m_{\alpha}(\mathscr{U}) \leq \varepsilon_{0}$ then every $U \in \mathscr{U}$ intersects at most one $K_{J}^{s},(s, J) \in \mathscr{J}$. Since $H D(\Lambda \cap[p, x])<\alpha$ there is such an $\mathcal{U}$. Denote $\mathcal{U}_{J}^{s}=\left\{U \in \mathscr{U}: U \cap K_{J}^{s} \neq \varnothing\right\}$. Then $\sum_{\mathscr{J}} m_{\alpha}\left(\mathcal{U}_{J}^{s}\right) \leq m_{\alpha}(U) \leq \varepsilon_{0}$, so there is $(t, L) \in \mathscr{J}, L=\left(l_{1}, \ldots, l_{n}\right)$, such that $m_{\alpha}\left(\mathcal{U}_{L}^{t}\right) \leq$ $\left(\lambda_{L}^{t} / r\right)^{\alpha} \varepsilon_{0}$. Form $\tilde{U}=\pi_{1}^{u}\left(H_{1} \cap f^{-|L|}\left(\mathcal{U}_{L}^{t}\right)\right)$ where $H_{1} \subset f^{-|L|}\left(K_{L}^{t}\right)$ and $\pi_{1}^{u}: H_{1} \rightarrow[p, x]$ are as at the beginning of the section. For every $U \in \mathcal{U}_{L}^{t}$ we have

$$
\operatorname{diam}\left(\pi_{1}^{u}\left(A_{1} \cap f^{-|L|}(U)\right)\right) \leq\left(r / \lambda_{L}^{t}\right) \cdot \operatorname{diam}(U),
$$

so $m_{\alpha}(\tilde{U}) \leq\left(r / \lambda_{L}^{t}\right)^{\alpha} m_{\alpha}\left(\mathcal{U}_{L}^{t}\right) \leq \varepsilon_{0}$. On the other hand $\tilde{\mathcal{U}}$ has strictly less elements than $\mathcal{U}$. Repeating this argument we get a covering of $\Lambda \cap[p, x]$ with no elements at all. This contradiction proves $H D(\Lambda \cap[p, x]) \geq \alpha_{n}$ for all $n \geq 1$.

Proof. of 5.1.b(ii). Suppose $d(\Lambda \cap[p, x])>\beta_{n}$ : then $\Lambda_{n}(d(\Lambda \cap[p, x]))<1$. Let $\beta>d(\Lambda \cap[p, x])>\alpha>\frac{1}{2}$ and take $A_{0}>0$ such that

$$
n(\varepsilon)=n(\Lambda \cap[p, x], \varepsilon) \leq A_{0} \cdot \varepsilon^{-\beta}
$$

for all $0<\varepsilon \leq 1$. If $U$ is a covering of $\Lambda \cap[p, x]$ by $\varepsilon$-balls with not more than $\left(A_{0} \varepsilon^{-\beta}\right)$ elements and $H_{i} \subset f^{-J \mid}\left(K_{J}^{s}\right)$ and $\pi_{i}^{u}: H_{i} \rightarrow[p, x]$ are as at the beginning of the section, then $\bigcup_{i=1}^{k} f^{|J|}\left(\left(\pi_{i}^{u}\right)^{-1}(\mathcal{U})\right)$ is a covering of $K_{J}^{s}$ by no more than $\left(k A_{0} \varepsilon^{-\beta}\right)$ intervals, each one of them contained in some $\left(\Lambda_{J}^{s} \cdot r \varepsilon\right)$-ball. Therefore

$$
n\left(K_{J}^{s}, \Lambda_{J}^{s} \cdot r \varepsilon\right) \leq k A_{0} \varepsilon^{-\beta}
$$


for $0<\varepsilon \leq 1$, i.e.,

$$
n\left(K_{J}^{s}, \xi\right) \leq k A_{0}\left(\Lambda_{J}^{s} \cdot r\right)^{\beta} \cdot \xi^{-\beta}
$$

if $0<\xi \leq r \Lambda_{j}^{s}$. Take, for every $0<\xi \leq 1$,

Then

$$
\mathscr{J}=\mathscr{J}(\xi)=\left\{(s, J): \xi \leq r \Lambda_{J}^{s}\right\}
$$

$$
n(\xi) \leq \sum_{\mathscr{J}} k A_{0}\left(\Lambda_{J}^{s} \cdot r\right)^{\beta} \cdot \xi^{-\beta}+n\left(\bigcup_{\mathscr{J}^{c}} K_{J}^{s}, \xi\right) .
$$

Claim. There is $A>0$ such that $n\left(\bigcup_{\mathscr{I}^{c}} K_{J}^{s}, \xi\right) \leq A \xi^{-\alpha}$ for all $0<\xi \leq 1$.

We postpone the proof of this claim and proceed to prove that it implies 5.1.b(ii).

Replacing above we get

$$
n(\xi) \leq A_{0} \Lambda_{n}(\beta) \cdot \xi^{-\beta}+A \cdot \xi^{-\alpha}=A_{1}(\xi) \cdot \xi^{-\beta}
$$

for all $0<\xi \leq 1$, where

$$
A_{1}(\xi)=A_{0} \Lambda_{n}(\beta)+A \cdot \xi^{\beta-\alpha}
$$

Repeating this argument we prove

$$
n(\xi) \leq A_{i}(\xi) \xi^{-\beta}
$$

for $0<\xi \leq 1$ and $i \geq 1$, where $\left(A_{i}(\xi)\right)_{i}$ is defined by

$$
A_{i+1}(\xi)=\Lambda_{n}(\beta) \cdot A_{i}(\xi)+A \cdot \xi^{\beta-\alpha}
$$

(and $A_{0}(\xi) \equiv A_{0}$ ).

Clearly $A_{i}(\xi) \geq A_{0}\left(\Lambda_{n}(\beta)\right)^{i}$. Let $\Lambda_{0}$ be such that

$$
\Lambda_{n}(d(\Lambda \cap[p, x]))<\Lambda_{0}<1
$$

and, given $0<\xi \leq 1$, take $j \geq 1$ minimum such that

$$
A \cdot \xi^{\beta-\alpha}>A_{0} \cdot\left(\Lambda_{0}-\Lambda_{n}(\beta)\right) \cdot \Lambda_{n}(\beta)^{j}
$$

By induction, $A_{i}(\xi) \leq A_{0} \cdot \Lambda_{0}^{i}$ for all $0 \leq i \leq j$ and so

It follows that

$$
n(\xi) \leq A_{0} \Lambda_{0}^{j} \cdot \xi^{-\beta} .
$$

$$
\begin{aligned}
{\left[\log n(\xi) / \log \xi^{-1}\right] \leq } & \beta+\left(\log A_{0} / \log \xi^{-1}\right)+\left(\log \Lambda_{0}^{j} / \log \xi^{-1}\right) \\
\leq & \beta-\left(\log A_{0} / \log \xi\right)-\left[(\beta-\alpha) \cdot\left(j \log \Lambda_{0}\right) /\left(j \log \Lambda_{n}(\beta)\right.\right. \\
& \left.\left.+\log \left(A_{0}\left(\Lambda_{0}-\Lambda_{n}(\beta)\right) / A\right)\right)\right] .
\end{aligned}
$$

Making $\xi \rightarrow 0$ (and so $j \rightarrow \infty$ ) we get

$$
d(\Lambda \cap[p, x]) \leq \beta-\left[(\beta-\alpha) \cdot \log \Lambda_{0} / \log \Lambda_{n}(\beta)\right]
$$

and this leads to a contradiction when $\beta \rightarrow d(\Lambda \cap[p, x])$.

To prove the claim it is sufficient to show that for some $c_{0}, \ldots, c_{n-1}$ and $b_{0}>0$ we have

$$
n\left(\bigcup_{\mathcal{F}^{\mathrm{c}}} K_{J}^{s}, \xi\right) \leq \xi^{-1 / 2}\left(\sum_{0}^{n-1} c_{i}\left(\log \left(b_{0} \xi^{-1 / 2}\right)\right)^{i}\right)
$$

Let

$$
\xi \geq r \Lambda_{J}^{s} \geq r a^{-1} \prod_{1}^{n}\left(\frac{1}{d j_{i}^{2}}\right)
$$


Suppose first

$$
\xi \geq r a^{-1}\left(\frac{1}{d j_{1}^{2}}\right) \text {. }
$$

Then, by the estimates in Lemma 5.2, $\Delta_{j_{1}} \leq c_{0} \sqrt{\xi}$ where $c_{0}=e \sqrt{a d / r}$. This means that such $K_{J}^{s}$ are contained in the $c_{0} \sqrt{\xi}$-ball centered at $p$ and so their union may be covered by $c_{0} \xi^{-1 / 2} \xi$-balls. In general take $k$ minimum such that

$$
\xi \geq r a^{-1} \prod_{1}^{k}\left(\frac{1}{d j_{i}^{2}}\right)
$$

Then, again by Lemma 5.2 ,

$$
\Delta_{j_{1}, \ldots, j_{k}}^{s} \leq b_{k} \sqrt{\xi} /\left(j_{1} \cdots j_{k-1}\right)
$$

where $b_{k}=e \sqrt{a d^{3 k-2} / r}$, and so these $K_{J}^{s}$ are contained in the $b_{k} \sqrt{\xi} /\left(j_{1} \cdots j_{k-1}\right)$-ball centered at the corresponding $p_{j_{1}, \ldots, j_{k-1}}$. Note that, by definition of $k$,

$$
\xi \leq r a^{-1} \Pi_{1}^{k-1}\left(\frac{1}{d j_{i}^{2}}\right) \leq \frac{r a^{-1}}{d j_{i}^{2}}
$$

for all $1 \leq i \leq k-1$. Therefore the union of such $K_{J}^{s}$ can be covered by not more than $\left(b_{k} \xi^{-1 / 2} \sum 1 / j_{1} \cdots j_{k-1}\right) \xi$-balls, where the sum is taken on the set of $j_{i}, 1 \leq i \leq k-1$, with $j_{i} \leq \sqrt{r /(a d \xi)}$. Now

$$
\sum\left[1 /\left(j_{1} \cdots j_{k-1}\right)\right]=\left(\sum_{1}^{\sqrt{r / a d \xi}} 1 / j\right)^{k-1} \leq\left(c \log \left(b_{0} \xi^{-1 / 2}\right)\right)^{k-1}
$$

for some universal $c>0$ and $b_{0}=\sqrt{r / a d}$. Repeating the argument for every $1 \leq k \leq n-1$ we get

$$
n\left(\bigcup_{\mathscr{F}^{c}} K_{J}^{s}, \xi\right) \leq \xi^{-1 / 2} \sum_{i=0}^{n-1}\left(c_{i}\left(\log \left(b_{0} \xi^{-1-/ 2}\right)\right)^{i}\right)
$$

with $c_{i}=c^{i-1} b_{i} l_{0}^{i-1}$ for $i \geq 1$.

Proof of 5.1.b(iii). Let $\delta_{n}=-\left(\log k\left(b r^{2}\right)^{\alpha_{n}} / \log \left(\lambda_{0}^{n-1} r\right)\right)$. Then

$$
\begin{aligned}
\Lambda_{n}\left(\alpha_{n}+\delta_{n}\right) & =k \sum_{s, J}\left(\Lambda_{J}^{s} r\right)^{\alpha_{n}+\delta_{n}} \leq k\left(\lambda_{0}^{n-1} r\right)^{\delta_{n}} \sum_{s, J}\left(\Lambda_{J}^{s} r\right)^{\alpha_{n}} \\
& \leq k\left(\lambda_{0}^{n-1} r\right)^{\delta_{n}}\left(b r^{2}\right)^{\alpha_{n}} \sum_{s, J}\left(\lambda_{J}^{s} / r\right)^{\alpha_{n}}=1 .
\end{aligned}
$$

Since $\Lambda_{n}$ is decreasing it follows $\beta_{n} \leq \alpha_{n}+\delta_{n}$. As $\left(\alpha_{n}\right)_{n}$ is bounded (by $H D(\Lambda[p, x])$ ) this ends the proof of 5.1.b(iii) and so of Proposition 5.1.

From the proposition it also follows $H D^{s}\left(\Lambda_{0}\right)=d^{s}\left(\Lambda_{0}\right)>1 / 2$. Note that $d\left(\Lambda_{0} \cap[p, x]\right) \geq \frac{1}{2}$ is a simple consequence of the fact that dist $\left(f^{j}(x), p\right)$ is of order $1 / j$ (Lemmas 3.2 and 5.2) together with $d(\{1 / j: j \geq 1\} \cup\{0\})=\frac{1}{2}$ (see Takens [11]).

Remark 5.3. The hypothesis that $p$ is a quadratic saddle-node for $f$ is not fundamental. If we assume that $k \geq 2$ is the order of the first non-degenerate jet of $f$ at the saddle-node then Lemmas 3.2 and 5.2 can be easily modified to give estimates for $l\left(K_{J}^{s}\right)$ and $\Delta_{J}^{s}$ similar to the ones above. In particular one gets

$$
H D\left(\Lambda_{0} \cap[p, x]\right)=d\left(\Lambda_{0} \cap[p, x]\right)>\frac{k-1}{k} .
$$


6. $H D^{2}\left(\Lambda_{t}\right) \rightarrow H D^{s}\left(\Lambda_{0}\right)$ as $t \rightarrow 0^{+}$

We show that the result $H D^{s}\left(\Lambda_{0}\right)=\lim _{n} \alpha_{n}=\lim _{n} \beta_{n}$ obtained in $\S 5$ can be extended to $H D^{s}\left(\Lambda_{t}\right)=\lim _{n} \alpha_{n}(t)=\lim _{n} \beta_{n}(t)$ for small positive $t$, where $\alpha_{n}(t), \beta_{n}(t)$ are constructed below. These functions turn out to be continuous at zero and this implies that $H D^{s}\left(\Lambda_{t}\right)$ is continuous on the right at $t=0$. Since $\alpha_{n}(t), \beta_{n}(t)$ are natural extensions of $\alpha_{n}, \beta_{n}$ we just sketch their construction and go into details only when they differ from $\S 5$.

Let $\left(A_{j}(t)\right)_{j}$ be a family of $u$-rectangles for $f_{t}$ satisfying, for every $t \geq 0$, properties analogous to $\left(P_{1}\right)-\left(P_{3}\right)$ of $\S 3$, with $A_{j}(0)=A_{j}$ for all $j \geq 1$ and depending continuously on $t$. By a $u$-rectangle we mean here an open imbedded rectangle contained in $W^{u}\left(q_{t}\right)$ and bounded by $W^{u}\left(\theta_{t}\right), W^{u}\left(p_{t}\right)$ and two segments of $W^{s}\left(p_{t}\right)$. As in $\S 5$ we define $x_{t}, y_{t}, K_{j}(t), K_{J}^{s}(t), \mathscr{K}^{n}(t), \lambda_{J}^{s}(t), \Lambda_{J}^{s}(t), p_{J}^{s}(t)$ and $\Delta_{J, j}^{s}(t)$. Finally let $\sigma(t)=\left|D^{s} f_{t}\left(p_{t}\right)\right|$.

LEMMA 6.1. There are $a, b, d_{1}, d_{2}, e>0$ and $0<\lambda_{0}<1$ such that:

(a) $a^{-1} \lambda_{J}^{s}(t) \leq l\left(K_{J}^{s}(t)\right) \leq a \Lambda_{J}^{s}(t)$,

(b) $\Lambda_{J}^{s}(t) \leq b \lambda_{J}^{s}(t)$,

(c) $\Lambda_{J}^{s}(t) \leq \lambda_{0}^{n-1}$,

(d $\left.\mathrm{d}_{1}\right) l\left(K_{J}^{s}(t)\right) \leq \Pi_{i}^{n} d_{1} / j_{i}^{2}$,

$\left(\mathrm{d}_{2}\right) l\left(K_{J}^{s}(t)\right) \leq d_{2}^{n} \sigma(t)$,

(e) $\Delta_{J, j}^{s}(t) \leq(e / j) \Pi_{1}^{n} d / j_{i}^{2}$,

for all $n, J=\left(j_{1}, \ldots, j_{n}\right), j$, s and $t>0$ small.

Proof. (a) and (c) are proved as in Lemma 5.2. The same holds for (b) once we have extended Lemma 3.3. for $t \geq 0$, (which is straightforward), with constant $c>0$ independent of $t$. This is possible because Lipschitz constants for $\mathscr{F}_{t}^{u}$ holonomies may be taken uniformly (see Remark 2.2).

To prove $\left(d_{1}\right)$ let $\varphi_{t}:[0,+\infty) \rightarrow\left\{p_{t}\right\} \cup W_{t}$ be the parametrization by arc-length, $\tilde{x}_{t}=\varphi_{t}^{-1}\left(x_{t}\right)$ and $\tilde{f}_{t}=\varphi_{t}^{-1} \circ f_{t} \circ \varphi_{t}$. Take $t_{0}>0, x_{0}>0$ and $a_{1}>0$ such that

$$
\sigma(t) x-2 a_{1} x^{2} \leq \tilde{f}_{t}(x) \leq \sigma(t) x-a_{1} x^{2}
$$

for $0 \leq x \leq x_{0}$ and $0 \leq t \leq t_{0}$. Take $m \geq 1$ such that $\tilde{f}_{t}^{m}\left(\tilde{x}_{t}\right) \leq\left(x_{0} / 2\right)$ for $0 \leq t \leq t_{0}$ and $m a_{1} x_{0} \geq 1$ and let

$$
a_{2}=\max \left\{a_{1}^{-1}, \sup \left\{\tilde{f}_{t}^{m}\left(\tilde{x}_{t}\right) \sum_{i=0}^{m-1} \sigma^{-1}(t), 0 \leq t \leq t_{0}\right\}\right\} .
$$

By induction we get, for $j \geq m$,

$$
\tilde{f}_{t}^{j}\left(\tilde{x}_{t}\right) \leq a_{2} \sigma(t)^{j-1} /\left(\sum_{0}^{j-1} \sigma(t)^{i}\right) .
$$

Then

$$
\begin{aligned}
l\left(K_{j+1}(t)\right) & \leq \tilde{f}_{t}^{j}\left(\tilde{x}_{t}\right)-\tilde{f}_{t}^{j+1}\left(\tilde{x}_{j}\right) \\
& \leq\left[a_{2} \sigma(t)^{j-1}(1-\sigma(t)) \sum_{0}^{j-1} \sigma(t)^{i}+2 a_{1} a_{2}^{2} \sigma(t)^{2 j-2}\right] /\left(\sum_{0}^{j-1} \sigma(t)^{i}\right)^{2} \\
& \leq a_{3} \sigma(t)^{j-1} /\left(\sum_{0}^{j-1} \sigma(t)^{i}\right)^{2} \leq a_{3} / j^{2}
\end{aligned}
$$


for some $a_{3}>0$ and all $j \geq m$. This proves case $n=1$ in $\left(d_{1}\right)$. The inductive step goes as in the proof of Lemma 5.2(d).

Case $n=1$ in $\left(d_{2}\right)$ and $n=0$ in (e) are weaker versions of estimates in the proof of $\left(d_{1}\right)$. The inductive step goes again as in Lemma 5.2. The proof of Lemma 6.1 is complete.

Let, as in $\S 5$,

and

$$
\lambda_{n}(t, \gamma)=\sum_{s, J}\left(\lambda_{J}^{s}(t) / r\right)^{\gamma}
$$

$$
\Lambda_{n}(t, \gamma)=k \sum_{s, J}\left(r \Lambda_{J}^{s}(t)\right)^{\gamma}
$$

where $r$ is some uniform Lipschitz constant for $\mathscr{F}_{t}^{u}$ holonomies.

\section{Proposition 6.2}

(a) For $n \geq 1$ big enough, $\lambda_{n}(t, \cdot)$ and $\Lambda_{n}(t, \cdot)$ are finite, continuous and strictly decreasing functions in $(0,+\infty)$. Moreover $\lim _{\gamma \rightarrow+\infty} \Lambda_{n}(t, \gamma)=\lim _{\gamma \rightarrow+\infty} \Lambda_{n}(t, \gamma)=$ 0 and $\lim _{\gamma \rightarrow 0+} \lambda_{n}(t, \gamma)=\lim _{y \rightarrow \infty^{+}} \Lambda_{n}(t, \gamma)=+\infty$.

(b) Let, for $n$ big $0<\alpha_{n}(t) \leq \beta_{n}(t)<+\infty$ be such that $\lambda_{n}\left(t, \alpha_{n}(t)\right)=1=\Lambda_{n}\left(t, \beta_{n}(t)\right)$. Then:

(i) $H D^{s}\left(\Lambda_{t}\right) \geq \alpha_{n}(t)$,

(ii) $d^{s}\left(\Lambda_{t}\right) \leq \beta_{n}(t)$,

(iii) $\left(\beta_{n}(t)-\alpha_{n}(t)\right)_{n} \rightarrow 0$.

Proof. All the affirmatives in (a) proved as in Proposition 5.1 except for the last one (which here is trivial). The same holds for $b(i)$ and $b(i i i)$. Finally $b$ (ii) also follows as in Proposition 5.1, once we have shown that $d^{s}\left(\Lambda_{t}\right)>\frac{1}{2}$, which is a consequence of 5.1, 6.2.b(i) and the proposition below.

PROPOSITION 6.3. For $n$ big $\alpha_{n}(t) \rightarrow \alpha_{n}$ and $\beta_{n}(t) \rightarrow \beta_{n}$ as $t \rightarrow 0^{+}$.

Proof. Let $\varepsilon>0$ : then $\sum_{s, J}\left(\lambda_{J}^{s} / r\right)^{\alpha_{n}-\varepsilon}>1$. Take $\Phi$ finite and $\delta>0$ such that $\sum_{\mathscr{f}}\left(\lambda_{J}^{s}(t) / r\right)^{\alpha_{n}-\varepsilon}>1$ for all $0 \leq t \leq \delta$. Then $\lambda_{n}\left(t, \alpha_{n}-\varepsilon\right)>1$, and so $\alpha_{n}(t)>\alpha_{n}-\varepsilon$. On the other hand $\sum_{s, J}\left(\lambda_{J}^{s} / r\right)^{\alpha_{n}+\varepsilon}=1-\tau$ for some $\tau>0$. Take $\mathscr{J}$ finite such that $\sum_{\mathscr{J}^{c}}\left(\lambda_{J}^{s}(t) / r\right)^{\alpha_{n}+\varepsilon}<\tau / 2$ for all $t$ close to zero (recall Lemma 6.1(a) and specially $\left.\left(d_{1}\right)\right)$. Now, if $t$ is small enough, $\sum_{g}\left(\lambda_{J}^{s}(t) / r\right)^{\alpha_{n}+\varepsilon}<1-\tau / 2$ and so $\lambda_{n}\left(t, \alpha_{n}+\varepsilon\right)<1$, which implies $\alpha_{n}+\varepsilon>\alpha_{n}(t)$. The proof for $\beta_{n}(t)$ is the same. This proves the proposition.

Continuity of $H D^{s}\left(\Lambda_{t}\right)$ and $d^{s}\left(\Lambda_{t}\right)$ as $t \rightarrow 0^{+}$now follows easily.

\section{Proof of Theorem $B^{\prime}$}

Let a defining sequence for a Cantor set $K \subset \mathbf{R}$ be a decreasing family $\left(K^{n}\right)_{n \geq 0}$ of compact parts of $\mathbf{R}$ with $K=\bigcap_{0}^{\infty} K^{n}$, such that $K^{0}$ is an interval and for $n \geq 1$ each $K^{n-1}$-component is a finite disjoint union of $K^{n}$-components and open intervals, the $K^{n}$-gaps. In the proof of Theorem $B^{\prime}$ we use the lower bound for the Hausdorff dimension biven by

LeMMA 7.1. Let $\tau>0$ and $K$ be a Cantor set with a defining sequence $\left(K^{n}\right)_{n}$ such that $[l(C)) / l(G)] \geq \tau$ for all $K^{n}$-component $C$ and $K^{n}$-gap $G$ with $\partial C \cap \partial G \neq \varnothing$, and all $n \geq 0$. Then $H D(K) \geq \log 2 / \log \left(2+\tau^{-1}\right)$. 
Proof. We need the following result whose (elementary) proof we leave to the reader: Let $\tau>0$ and $\beta \leq \log 2 / \log \left(2+\tau^{-1}\right)$. Then

$$
x+y+z=a, x \geq \tau y, z \geq \tau y \text { with } x, y, z>0 \Rightarrow x^{\beta}+z^{\beta} \geq a^{\beta} .
$$

Let $\beta=\log 2 / \log \left(2+\tau^{-1}\right)$. We prove that for all finite open covering $\mathcal{U}$ of $K$ we have $m_{\beta}(U) \geq\left(\operatorname{diam} K^{0}\right)^{\beta}$. We may assume that $U$ is disjoint, since if $U_{1}, U_{2} \in \mathcal{U}$ have non-empty intersection then $\left(l\left(U_{1} \cup U_{2}\right)\right)^{\beta} \leq\left(l\left(U_{1}\right)\right)^{\beta}+\left(l\left(U_{2}\right)\right)^{\beta}$, which means that the covering obtained from $\mathcal{U}$ replacing $U_{1}, U_{2}$ by $U_{1} \cup U_{2}$ has smaller $\beta$-measure than $U$. Let now $n \geq 0$ be the smallest integer such that $U$ covers $K^{n}$. Let $G$ be a $K^{n}$-gap not covered by $U$ and $C_{l}, C_{r}$ by the $K^{n}$-components with $\partial C_{l} \cap \partial G \neq \varnothing \neq \partial G \cap \partial C_{r}$. Denote by $U_{l}, U_{r}$ the elements of $\mathscr{U}$ containing $C_{l}$ and $C_{r}$ respectively and let $U$ be their convex hull and $V=U-\left(U_{i} \cup U_{r}\right)$. Then

$$
l\left(U_{l}\right) \geq l\left(C_{l}\right), l\left(U_{r}\right) \geq l\left(C_{r}\right), l(V) \leq l(G)
$$

which implies $l\left(U_{l}\right) \geq \tau \cdot l(V), l\left(U_{r}\right) \geq \tau \cdot l(V)$ and so $\left.\left(l\left(U_{l}\right)\right)^{\beta}+\left(l\left(U_{r}\right)\right)^{\beta} \geq l(U)\right)^{\beta}$. This means that replacing $U_{l}, U_{r}$ by $U$ in $\mathcal{U}$ we get a covering of $K$ with smaller $\beta$-measure than $\mathcal{U}$. Repeating the argument we construct a covering $\mathscr{V}$ of $K^{0}$ with $m_{\beta}(\mathscr{V}) \leq m_{\beta}(\mathscr{U})$. Since we must have $m_{\beta}(\mathscr{V}) \geq\left(\operatorname{diam} K^{0}\right)^{\beta}$ this proves the lemma.

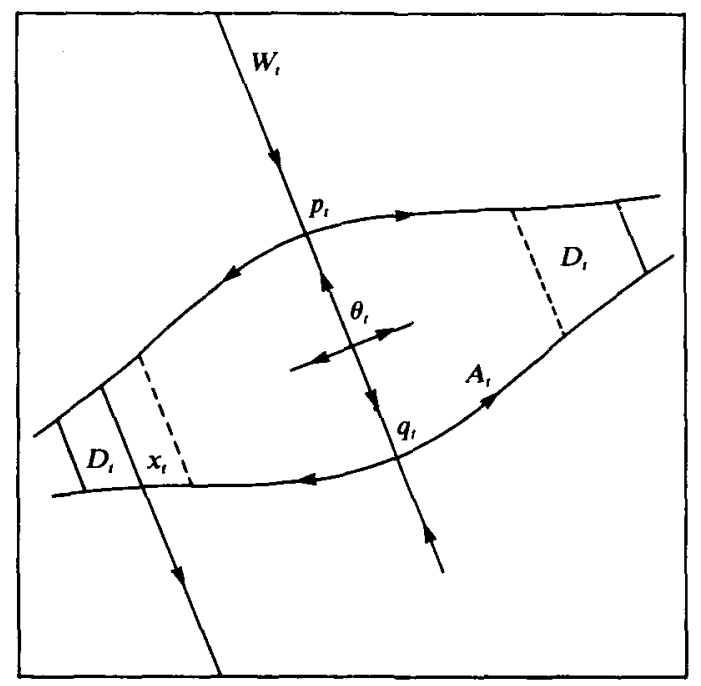

FIGURE 5

We now prove Theorem $B^{\prime}$. Take, for $t>0, A_{t}$ a $u$-rectangle (i.e. an open domain contained in $W^{u}\left(\theta_{t}\right)$ and bounded by $W^{u}\left(p_{t}\right), W^{u}\left(q_{t}\right)$ and two segments of $\left.W^{s}\left(p_{t}\right)\right)$ containing $\theta_{t}$ and let $D_{t}=A_{t}-f_{t}^{-1}\left(A_{t}\right)$ be a fundamental domain for $W^{u}\left(\theta_{t}\right)$. Let $W_{t}$ be the separatrix of $W^{s}\left(p_{t}\right)-\left\{p_{t}\right\}$ not contained in $W^{u}\left(\theta_{t}\right)$ and let $x_{t} \in W_{t} \cap W^{u}\left(q_{t}\right)$ be the smallest element of $W_{t} \cap \partial^{u} A_{t}$ (for the natural ordering in $W_{t}$, see $\left.\S 3\right)$.

Define $K_{t}^{0}=\left[p_{t}, x_{t}\right]$ and, for $n \geq 1, K_{t}^{n}=K_{t}^{0}-f_{t}^{n}\left(A_{t}\right)=K_{t}^{n-1}-f_{t}^{n}\left(D_{t}\right)$. Then $\Lambda_{t} \cap$ $\left[p_{t}, x_{t}\right]=\bigcap_{0}^{\infty} K_{t}^{n}$ and $\left(K_{t}^{n}\right)_{n}$ is a defining sequence for $\Lambda_{t} \cap\left[p_{t}, x_{t}\right]$. Note that $C$ is 
a $K_{t}^{n}$-component if and only if $C=f_{t}^{n}\left(C_{0}\right) \subset\left[p, x_{t}\right]$ for some connected component $C_{0}$ of $W_{t}-A_{t}$. Also the $K_{t}^{n}$-gaps are the $G=f_{t}^{n}\left(G_{0}\right) \subset\left[p_{t}, x_{t}\right], G_{0}$ a connected component of $W_{t} \cap D_{t}$.

Claim. There is $a>0$ such that $\left[l\left(f^{n}\left(C_{0}\right)\right) / l\left(f^{n}\left(G_{0}\right)\right)\right] \geq a\left[l\left(C_{0}\right) / l\left(G_{0}\right)\right]$ for all component $C_{0}$ of $W_{t}-A_{t}$, all component $G_{0}$ of $W_{t} \cap D_{t}$ with $\partial C \cap \partial G \neq \varnothing$, and all $n \geq 1$ and $t>0$.

Take then $b(t)=\inf \left\{l\left(C_{0}\right) / l\left(G_{0}\right): C_{0}, G_{0}\right.$ as above $\}$. Clearly $b(t)>0$ and $b(t) \rightarrow$ $+\infty$ as $t \rightarrow 0^{+}$. From the claim we get $[l(C) / l(G)] \geq a b(t)$ for all $K_{t}^{n}$-component $C$ and $K_{t}^{n}$-gap $G$ with $\partial C \cap \partial G \leq \varnothing$. Theorem $\mathrm{B}^{\prime}$ now follows from Lemma 7.1.

We are now left to prove the claim above. Let $W_{t}^{\prime}$ be the separatrix of $W^{s}\left(q_{t}\right)-\left\{q_{t}\right\}$ not contained in $W^{u}\left(\theta_{t}\right)$ and let $z_{t} \in W_{t}^{\prime} \cap W^{u}\left(p_{t}\right)$ be the smallest element of $W_{t}^{\prime} \cap \partial^{\prime \prime} A_{t}$ (for the natural ordering in $W_{t}^{\prime}$, see $\S 3$ ).

Take $y_{t} \in W_{t} \cap W^{u}\left(p_{t}\right)$ and $w_{t} \in W_{t}^{\prime} \cap W^{u}\left(q_{t}\right)$ such that $\left[x_{t}, y_{t}\right]$ and $\left[z_{r}, w_{t}\right]$ are connected components of $W_{t} \cap A_{t}$ and $W_{t}^{\prime} \cap A_{t}$ respectively. Let $B_{t}$ and $C_{t}$ be u-rectangles intersecting all the connected components of $W_{t}-A_{t}$ and such that $B_{t} \cap\left[p_{t}, x_{t}\right]=\left[f_{t}\left(y_{t}\right)\right]$ and $C_{t} \cap\left[q_{t}, z_{t}\right]=\left[f_{t}\left(z_{t}\right), f_{t}\left(\omega_{t}\right)\right]$. (See figure 6.)

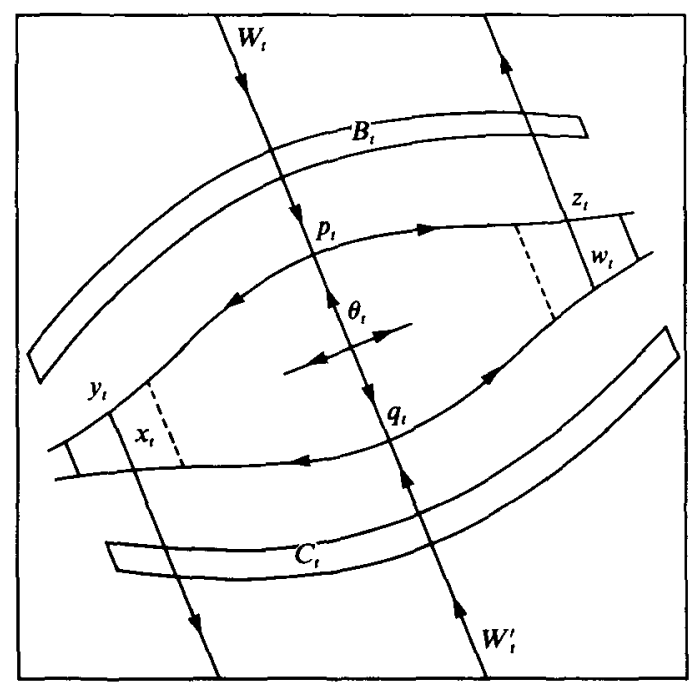

Figure 6

LEMMA 7.2. (Distortion Lemma). There is $c>0$ such that $\sum_{0}^{\infty} l\left(f_{t}^{n}\left(G_{0} \cup H_{0}\right)\right) \leq c$ for every connected component $G_{0}$ of $W_{t} \cap D_{t}$, every connected component $H_{0}$ of $W_{t}-\left(A_{t} \cup B_{t} \cup C_{t}\right)$ with $\partial H_{0} \cap \partial G_{0} \neq \varnothing$ and all $t>0$.

Lemma 7.2 is proved in almost the same way as Lemma 3.3 so we do not go into details here. We just note that, by construction, iterates of $\left(G_{0} \cup H_{0}\right)$ passing near $p_{t}$ (resp. $q_{t}$ ) project into disjoint intervals in $W_{t}\left(\right.$ resp. $\left.W_{t}^{\prime}\right)$ and this permits the same kind of estimates for the sum of the lengths of those iterates as in the proof of Lemma 3.3 (see also the remark at the end of this section). We should also point out that, in order to get $c>0$ independent of $t>0$, one must use the fact that 
Lipschitz constants for the unstable holonomy $\pi^{u}$ may be taken uniform on $t$ (Remark 2.2).

Let now $C_{0}$ and $G_{0}$ be as in the statement of the claim. Take $H_{0}$ a connected component of $W_{t}-\left(A_{t} \cup B_{t} \cup C_{t}\right)$ contained in $C_{0}$, with $\partial G_{0} \cap \partial H_{0} \neq \varnothing$. Then, for some $\xi \in G_{0}$ and $\eta \in H_{0}$, we have

$$
\begin{aligned}
& {\left[l\left(f^{n}\left(C_{0}\right)\right) / l\left(f^{n}\left(G_{0}\right)\right)\right] \geq\left[l\left(f^{n}\left(H_{0}\right)\right) / l\left(f^{n}\left(G_{0}\right)\right)\right]} \\
& \left.\quad=\left[l\left(H_{0}\right) / l\left(G_{0}\right)\right] \cdot\left[\mid D^{s} f_{t}^{n}\right) /\left|D f_{t}^{n}(\eta)\right|\right] \\
& \quad=\left[l\left(C_{0}\right) / l\left(G_{0}\right)\right] \cdot\left[l\left(H_{0}\right) / l\left(C_{0}\right)\right] \exp \left(\sum_{0}^{n-1} \log \left|D^{s} f_{t}\right|\left(f^{j} \xi\right)-\log \left|D^{s} f_{t}\right|\left(f^{j} \eta\right)\right) \\
& \quad \geq\left[l\left(C_{0}\right) / l\left(G_{0}\right)\right] \cdot a_{1} \cdot \exp \left(-\operatorname{Lip}\left(\log \left|D^{s} f_{t}\right|\right) \cdot \sum_{0}^{n-1} d\left(f^{j} \xi, f^{j} \eta\right)\right) \\
& \quad \geq a_{1} \cdot \exp \left(-c \cdot \operatorname{Lip}\left(\log \left|D^{s} f_{t}\right|\right)\right) \cdot\left[l\left(C_{0}\right) / l\left(G_{0}\right)\right],
\end{aligned}
$$

where $c>0$ is given by Lemma 7.2 and $a_{1} \geq 0$ is some uniform lower bound for $\left(l\left(H_{0}\right) / l\left(C_{0}\right)\right)$, with $H_{0}$ and $C_{0}$ as above. Since $\left(f_{t}\right)_{t}$ is continuous in the $C^{2}$-topology $\operatorname{Lip}\left(\log \left|D^{s} f_{t}\right|\right)$ is bounded, so we may take $a=a_{1} \exp \left(-c \sup _{t} \operatorname{Lip}\left(\log \left|D^{s} f_{t}\right|\right)\right)$. This ends the proof of our claim and thus of the theorem.

Remark 7.3. $\sum_{0}^{\infty} l\left(f_{t}^{n}\left(C_{0} \cup G_{0}\right)\right), C_{0}$ a component of $\left(W_{t}-A_{t}\right)$ and $G_{0}$ a component of $\left(W_{t} \cap D_{t}\right)$ with $\partial C_{0} \cap \partial G_{0} \neq \varnothing$, is not uniformly bounded. For example, it is not hard to see that $\sum_{0}^{\infty} l\left(f_{i}^{n}\left(\left[p_{t}, x_{i}\right]\right)\right) \rightarrow+\infty$ as $t \rightarrow 0^{+}$. Note that $\left(f_{t}^{n}\left(\left[p_{t}, x_{i}\right]\right)\right)_{n}$ is not disjoint.

\section{Proof of Theorem $C$}

We first claim that $\lim \mu_{t}\left(\phi_{t}^{s}\right) \rightarrow 0$ as $t \rightarrow 0^{+}$. In fact by the variational principle $P\left(\alpha \phi_{t}^{s}\right) \geq h_{\mu_{t}}\left(f_{t}\right)+\alpha \mu_{t}\left(\phi_{t}^{s}\right), \alpha \geq 0$, with equality for $\alpha=1$, and by Theorem 1 in [4] we have $P\left(H D^{s}\left(\Lambda_{t}\right) \phi_{t}^{s}\right)=0$ (see figure 7 ). Then noting that $\phi_{t}^{s}<0$ on $\Lambda_{t}$ we get

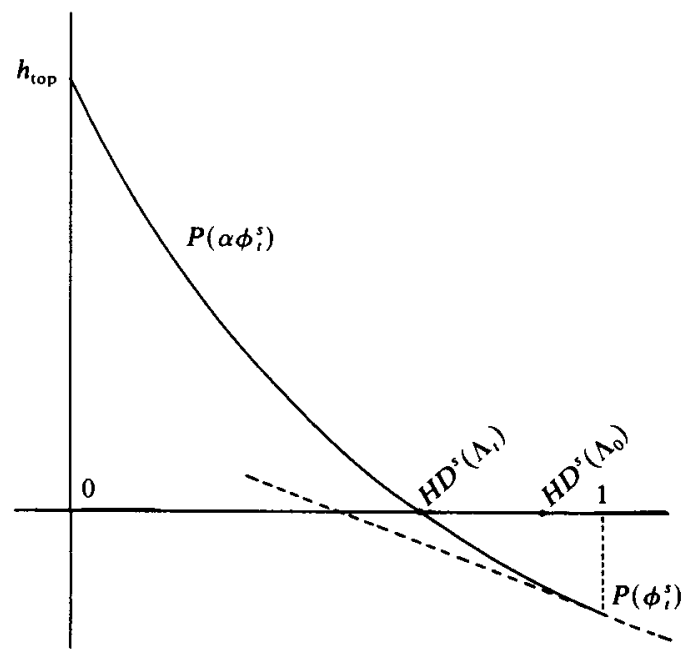

FIGURE 7 
$0 \geq \mu_{t}\left(\phi_{t}^{s}\right) \geq P\left(\phi_{t}^{s}\right) /\left(1-H D^{s}\left(\Lambda_{t}\right)\right)$. Since $H D^{s}\left(\Lambda_{t}\right) \rightarrow H D^{s}\left(\Lambda_{0}\right)<1$ as $t \rightarrow 0^{+}$the claim now follows from the fact that $P\left(\phi_{t}^{s}\right) \rightarrow 0$ as $t \rightarrow 0^{+}$. This was done in [4] but for the sake of completeness we reproduce the argument here. Let $\delta_{t}$ be the Dirac measure at the fixed point $p_{t}$. Clearly $h_{\delta_{t}}\left(f_{t}\right)=0$ and $\delta_{t}\left(\phi_{t}^{s}\right)=\phi_{t}^{s}\left(p_{t}\right)$ so (see also Theorem 4.11 in [1]) we have $0>P\left(\phi_{t}^{s}\right) \geq \phi_{t}^{s}\left(p_{t}\right) \rightarrow 0$ as $t \rightarrow 0^{+}$. Now the claim, together with $\phi_{t}^{s}<0$ on $\Lambda_{t}$, easily implies $\mu_{t} \rightarrow \delta$ as $t \rightarrow 0^{+}$.

To show that $\delta$ is the unique equilibrium state for $\phi_{0}^{s}$ we first note that

$$
0=h_{\delta}\left(f_{0}\right)+\delta\left(\phi_{0}^{s}\right) \leq P\left(\phi_{0}^{s}\right)=\sup \left\{h_{\mu}\left(f_{0}\right)+\mu\left(\phi_{\mu}^{s}\right): \mu \quad f \text {-invariant }\right\} \leq 0
$$

(see Ruelle [9] for the last inequality).

Now if $\mu$ is an equilibrium state for $\phi_{0}^{s}$ we must have $\mu\left(\phi_{0}^{s}\right)=0$ and so $\mu=\delta$. Otherwise by Manning [5] we would have

$$
H D^{s}\left(\Lambda_{0}\right) \geq H D\left(W_{\text {loc }}^{s}(p) \cap G_{\mu}\right) \geq-h_{\mu}\left(f_{0}\right) / \mu\left(\phi_{0}^{s}\right)=1
$$

(where $G_{\mu}$ is the set of generic points for $\mu$ ) and this contradicts Theorem A'(a). We should note that, although Manning's theorem is stated for Axiom A diffeomorphisms, the proof of the inequality we use here is more general and still applies in our context.

\section{REFERENCES}

[1] R. Bowen. Equilibrium states and ergodic theory of Axiom A diffeomorphisms. Lecture Notes in Mathematics 470. Springer Verlag: New York, 1975.

[2] R. Bowen. On Axiom A diffeomorphisms. Conference Board Math. Sciences 35 Amer. Math. Soc. (1977).

[3] M. Hirsch \& C. Pugh. Stable manifolds and hyperbolic sets. Global Analysis. Proc. Symp. in Pure Math. XIV, Amer. Math. Soc. (1970), 133-163.

[4] H. McCluskey \& A. Manning. Hausdorff dimension for horseshoes. Ergod. Th. \& Dynam. Sys. 3 (1983), 231-260.

[5] A. Manning. A relation between Lyapunov exponents, Hausdorff dimension and entropy. Ergod. Th. \& Dynam. Sys. 1 (1981), 451-459.

[6] A. Manning. Errata to 'Hausdorff dimension for horseshoes'. Ergod. Th. \& Dynam. Sys. 5 (1985), 319.

[7] J. M. Marstrand. The dimension of cartesian products of sets. Proc. Cambridge Phil. Soc. 50 (1954), 198-202.

[8] J. Palis \& M. Viana. On the continuity of Hausdorff dimensions and limit capacity for horseshoes. Topics in Dynamics, Proceedings Chilean Symp., Lecture Notes in Math. 1331. Springer Verlag: New York, 1988.

[9] D. Ruelle. An inequality for the entropy of differentiable maps. Bol. Soc. Bras. de Mat. 9 (1978), 83-87.

[10] S. Smale. Differentiable dynamical systems. Bull. Amer. Math. Soc. 73 (1967), 747-817.

[11] F. Takens. Limit capacity and Hausdorff dimension of dynamically defined Cantor sets. Topics in Dynamics, Proceedings Chilean Symp., Lecture Notes in Math. 1331. Springer Verlag: New York, 1988.

[12] P. Walters. A variational principle for the pressure of continuous transformations. Amer. J. Math. 97 (1976), 937-971.

[13] R. Williams. The 'DA' maps of Smale and structural stability. Global Analysis, Proc. Symp. in Pure Math. XIV Amer. Math. Soc. (1970), 329-334. 